IMA Journal of Applied Mathematics (2013) Page 1 of 35

doi: 10.1093/imamat/xxx000

\title{
Porous squeeze-film flow
}

\author{
D. J. KNOX*, S. K. WiLson $\dagger$, B. R. Duffy $¥$ And S. McKeE $§$ \\ Department of Mathematics and Statistics, University of Strathclyde, 26 Richmond Street, \\ Glasgow G1 1XH, United Kingdom
}

[Received on 25 January 2013, revised 26th August 2013]

\begin{abstract}
The squeeze-film flow of a thin layer of Newtonian fluid filling the gap between a flat impermeable surface moving under a prescribed constant load and a flat thin porous bed coating a stationary flat impermeable surface is considered. Unlike in the classical case of an impermeable bed, in which an infinite time is required for the two surfaces to touch, for a porous bed contact occurs in a finite contact time. Using a lubrication approximation an implicit expression for the fluid layer thickness and an explicit expression for the contact time are obtained and analysed. In addition, the fluid particle paths are calculated, and the penetration depths of fluid particles into the porous bed are determined. In particular, the behaviour in the asymptotic limit of small permeability, in which the contact time is large but finite, is investigated. Finally, the results are interpreted in the context of lubrication in the human knee joint, and some conclusions are drawn about the contact time of the cartilage-coated femoral condyles and tibial plateau and the penetration of nutrients into the cartilage.
\end{abstract}

Keywords: porous squeeze-film flow, finite contact time, fluid particle paths, penetration depths

\section{Introduction}

The squeeze-film flow of a thin layer of Newtonian fluid filling the gap between two impermeable surfaces is a classical problem in fluid mechanics (see, for example, Acheson (1990) and Szeri (1998)) and it is well known that under a prescribed constant load, at least in theory, an infinite time is required for two smooth surfaces to touch (see, for example, Stone (2005)). Of course, in practice, additional physical effects not included in the classical squeeze-film problem (such as, for example, small-scale surface roughness) will usually result in contact between the two surfaces in a finite (but possibly large) time. However, even in theory, there are situations in which contact can occur in a finite time. For example, recently Cawthorn \& Balmforth (2010) showed that a non-smooth body, namely a wedge falling apex-first under gravity, will make contact with an underlying surface in a finite time. In the present work we consider another situation in which, even in theory, contact occurs in a finite time, namely when one of the surfaces is porous.

Motivated in part by the fact that classical results were unable to describe the engagement of wet porous clutch plates, Wu (1970) considered the squeeze-film flow of a thin layer of fluid between two annular discs, the upper disc being covered with a porous layer. Wu (1970) used Reynolds' lubrication theory to describe the flow in the gap between the discs, and Darcy's law to describe the flow in the porous layer, and showed that the load-carrying capacity, i.e. the normal force exerted on the discs due to the fluid, is reduced in comparison with the case when both discs are impermeable. Prakash \& Vij (1973) used a similar model to describe the squeeze-film flow between circular, annular, elliptical and

\footnotetext{
*Email: darren.knox@ @trath.ac.uk

${ }^{\dagger}$ Author for correspondence. Tel: + 44 (0) 141548 3820, Email: s.k.wilson@ strath.ac.uk

\#Email: b.r.duffy@ strath.ac.uk

§Email: s.mckee@strath.ac.uk
} 


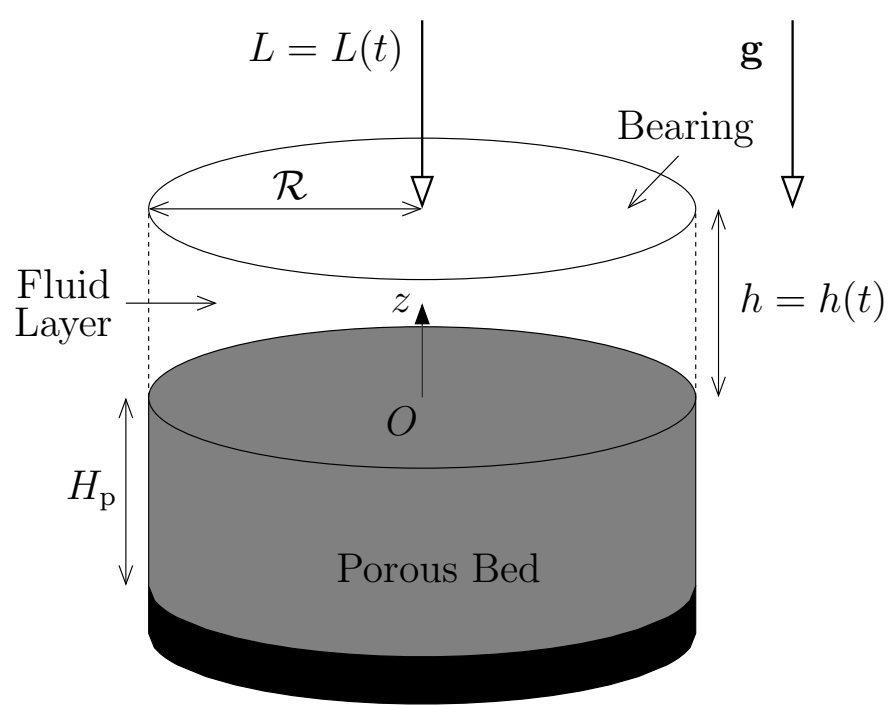

Rigid Impermeable Surface

FIG. 1. The geometry of the problem.

rectangular flat plates, the lower surface being covered with a porous layer; they also used a lubrication approximation for the flow in the porous layer and obtained results similar to those of Wu (1970).

In the present work we consider the squeeze-film flow of a thin layer of Newtonian fluid filling the gap between a flat impermeable surface moving under a prescribed constant load and a flat thin porous bed coating a stationary flat impermeable surface. Using a lubrication approximation an implicit expression for the fluid layer thickness and an explicit expression for the contact time are obtained and analysed. In addition, the fluid particle paths are calculated, and the penetration depths of fluid particles into the porous bed are determined. In particular, the behaviour in the asymptotic limit of small permeability, in which the contact time is large but finite, is investigated. Finally, the results are interpreted in the context of lubrication in the human knee joint, and some conclusions are drawn about the contact time of the cartilage-coated femoral condyles and tibial plateau and the penetration of nutrients into the cartilage.

\section{Problem Formulation}

\subsection{Problem Description}

Consider, with reference to the cylindrical polar coordinate system $(r, \theta, z)$ shown in Figure 1, the time-dependent axisymmetric flow in the thin cylindrical gap $0 \leqslant r \leqslant \mathscr{R}, 0 \leqslant z \leqslant h$ between a circular flat rigid impermeable bearing $z=h(t)$ of radius $\mathscr{R}$ moving vertically (i.e. in the $z$-direction) and a stationary circular flat rigid impermeable surface also of radius $\mathscr{R}$ at $z=-H_{\mathrm{p}}$, where $t$ denotes time. The rigid impermeable surface is coated with a thin cylindrical rigid porous bed of constant permeability $k$, porosity $\phi(0<\phi<1)$, depth $H_{\mathrm{p}}$ and radius $\mathscr{R}$ that occupies $0 \leqslant r \leqslant \mathscr{R},-H_{\mathrm{p}} \leqslant z \leqslant 0$. The gap between the bearing and the porous bed is filled with, and the pores in the porous bed are saturated 
with, an incompressible Newtonian fluid with constant density $\rho$ and viscosity $\mu$. We prescribe the load $L=L(t)>0$ that acts vertically downwards through the bearing, pushing it towards the porous bed.

The squeeze-film flow described here is axisymmetric, but the corresponding two-dimensional problem is also of some interest and is treated in Appendix A. In particular, as we show in Appendix A, the solutions for the fluid layer thickness $h=h(t)$ and the fluid particle paths $(x, y)$ may be readily obtained from the corresponding solutions in the axisymmetric case.

\subsection{Governing Equations and Boundary Conditions}

The axiymmetric flow in the fluid layer $(0 \leqslant r \leqslant \mathscr{R}, 0 \leqslant z \leqslant h)$ is governed by the mass-conservation and Navier-Stokes equations

$$
\nabla \cdot \mathbf{v}=0, \quad \rho\left(\frac{\partial \mathbf{v}}{\partial t}+(\mathbf{v} \cdot \nabla) \mathbf{v}\right)=-\nabla p+\mu \nabla^{2} \mathbf{v}+\rho \mathbf{g}
$$

where $\mathbf{v}=\left(v_{r}, 0, v_{z}\right)$ is the fluid velocity, with radial and vertical components $v_{r}=v_{r}(r, z, t)$ and $v_{z}=$ $v_{z}(r, z, t)$, respectively, $p=p(r, z, t)$ is the fluid pressure, and $\mathbf{g}=(0,0,-g)$ denotes (constant) acceleration due to gravity.

We assume that the axisymmetric flow in the porous bed $\left(0 \leqslant r \leqslant \mathscr{R},-H_{\mathrm{p}} \leqslant z \leqslant 0\right)$ is governed by the mass-conservation equation and Darcy's law,

$$
\nabla \cdot \mathbf{u}=0, \quad \mathbf{u}=-\frac{k}{\mu} \nabla P
$$

where $\mathbf{u}=\left(u_{r}, 0, u_{z}\right)$ is the so-called Darcy velocity (the fluid volume flux per unit area), with radial and vertical components $u_{r}=u_{r}(r, z, t)$ and $u_{z}=u_{z}(r, z, t)$, respectively, and $P=P(r, z, t)$ is the pore pressure. Combining (2.2) shows that $P$ is harmonic, i.e.

$$
\nabla^{2} P=0
$$

Assuming that the inertia of the bearing is negligible, the requirement that the prescribed load $L=$ $L(t)$ is equal to the net force exerted on the bearing by the fluid means that

$$
L=\left.2 \pi \int_{0}^{\mathscr{R}}\left(p-2 \mu \frac{\partial v_{z}}{\partial z}\right) r\right|_{z=h} \mathrm{~d} r
$$

On the interface between the bearing and the fluid layer at $z=h$ the no-slip and no-penetration conditions require that

$$
v_{r}=0, \quad v_{z}=\frac{\mathrm{d} h}{\mathrm{~d} t} \quad \text { on } \quad z=h .
$$

On the interface between the fluid layer and the porous bed at $z=0$ mass conservation requires that

$$
v_{z}=u_{z} \quad \text { on } \quad z=0,
$$

while continuity of normal stress requires that

$$
-p+2 \mu \frac{\partial v_{z}}{\partial z}=-P \quad \text { on } \quad z=0
$$


In addition, we impose the Beavers-Joseph condition

$$
\frac{k^{1 / 2}}{\alpha} \frac{\partial v_{r}}{\partial z}=v_{r}-u_{r} \quad \text { on } \quad z=0,
$$

where $\alpha(>0)$ is the dimensionless Beavers-Joseph constant (see the original work by Beavers \& Joseph (1967), the historical and critical note by Nield (2009), and the many papers using this condition, including, for example, Sherwood (1990), Wu (1972), Prakash \& Vij (1974), Lin et al. (2001), and Nong $\&$ Anderson (2010)). This condition allows for velocity slip on the interface between the fluid layer and the porous bed at $z=0$ with slip length $l_{\mathrm{s}}=k^{1 / 2} / \alpha$. In the limit $\alpha \rightarrow \infty$ the slip length $l_{\mathrm{s}} \rightarrow 0$ and (2.8) reduces to

$$
v_{r}=u_{r} \quad \text { on } \quad z=0,
$$

which corresponds to no slip relative to the fluid at the interface. If we neglect the radial Darcy velocity $u_{r}$ in (2.9) then the condition becomes

$$
v_{r}=0 \quad \text { on } \quad z=0,
$$

which corresponds to no slip relative to the stationary porous bed.

On the interface between the porous bed and the rigid impermeable surface at $z=-H_{\mathrm{p}}$ the nopenetration condition requires that

$$
u_{z}=0 \quad \text { on } \quad z=-H_{\mathrm{p}} .
$$

At $r=\mathscr{R}$ continuity of normal stress requires that the normal stress takes its ambient value, which we assume to be zero, i.e.

$$
-p+2 \mu \frac{\partial v_{r}}{\partial r}=0 \quad \text { at } \quad r=\mathscr{R},
$$

and, since the fluid pressure is axisymmetric and smooth at $r=0$, we also require that

$$
\frac{\partial p}{\partial r}=0 \quad \text { at } \quad r=0 .
$$

Finally, we must also specify the initial thickness of the fluid layer:

$$
h(0)=H_{\mathrm{f}}(>0) .
$$

In general, solving (2.1), (2.3) and (2.4) would require the specification of additional boundary and initial conditions. However, as is typical of problems of this type, when both the fluid layer and the porous bed are thin, and a lubrication approximation is used to describe the flow in both of them, the leading order approximations to these equations may, in fact, be solved without specifying any of these additional conditions.

\subsection{Lubrication Approximation}

We scale and non-dimensionalise the problem as follows:

$$
\begin{gathered}
r=\mathscr{R} r^{\prime}, \quad z=H_{\mathrm{p}} z^{\prime}, \quad t=\frac{\mathscr{R}}{V} t^{\prime}, \quad h=H_{\mathrm{p}} h^{\prime}, \quad k=H_{\mathrm{p}}^{2} k^{\prime}, \quad v_{r}=V v_{r}^{\prime}, \quad v_{z}=\frac{H_{\mathrm{p}} V}{\mathscr{R}} v_{z}^{\prime}, \\
u_{r}=V u_{r}^{\prime}, \quad u_{z}=\frac{H_{\mathrm{p}} V}{\mathscr{R}} u_{z}^{\prime}, \quad p=\frac{\mu V \mathscr{R}}{H_{\mathrm{p}}^{2}} p^{\prime}, \quad P=\frac{\mu V \mathscr{R}}{H_{\mathrm{p}}^{2}} P^{\prime}, \quad L=L_{0} L^{\prime},
\end{gathered}
$$


where a prime ' denotes a dimensionless variable and $\mathscr{R}, V=L_{0} H_{\mathrm{p}}^{2} /\left(\mu \mathscr{R}^{3}\right), \mu V \mathscr{R} / H_{\mathrm{p}}^{2}=L_{0} / \mathscr{R}^{2}$ and $L_{0}$ are characteristic scales for radial distance, radial fluid velocity, pressure and load, respectively.

In dimensionless variables, with the primes omitted for the sake of clarity, the mass-conservation and Navier-Stokes equations (2.1) become

$$
\begin{aligned}
\frac{1}{r} \frac{\partial}{\partial r}\left(r v_{r}\right)+\frac{\partial v_{z}}{\partial z} & =0 \\
R^{*}\left(\frac{\partial v_{r}}{\partial t}+v_{r} \frac{\partial v_{r}}{\partial r}+v_{z} \frac{\partial v_{r}}{\partial z}\right) & =-\frac{\partial p}{\partial r}+\frac{\partial^{2} v_{r}}{\partial z^{2}}+O\left(\varepsilon^{2}\right), \\
\varepsilon^{2} R^{*}\left(\frac{\partial v_{z}}{\partial t}+v_{r} \frac{\partial v_{z}}{\partial r}+v_{z} \frac{\partial v_{z}}{\partial z}\right) & =-\frac{\partial p}{\partial z}-G+O\left(\varepsilon^{2}\right),
\end{aligned}
$$

where the aspect ratio of both the fluid layer and the porous bed, $\varepsilon=H_{\mathrm{p}} / \mathscr{R} \ll 1$, the (reduced) Reynolds number for the flow in the fluid layer, $R^{*}=\varepsilon^{2} \rho V \mathscr{R} / \mu=\varepsilon^{2} \rho L_{0} H_{\mathrm{p}}^{2} /\left(\mu^{2} \mathscr{R}^{2}\right) \ll 1$, and the non-dimensional parameter that measures the relative importance of gravitational effects and viscous effects, $G=\varepsilon^{3} \rho g \mathscr{R}^{2} /(\mu V)=\varepsilon^{3} \rho g \mathscr{R}^{5} /\left(L_{0} H_{\mathrm{p}}^{2}\right) \ll 1$, are all assumed to be small. At leading order in the limit $\varepsilon \rightarrow 0$ equations (2.17) and (2.18) reduce to the classical lubrication equations

$$
\begin{aligned}
& \frac{\partial p}{\partial r}=\frac{\partial^{2} v_{r}}{\partial z^{2}} \\
& \frac{\partial p}{\partial z}=0 .
\end{aligned}
$$

Similarly, retaining higher order terms which will be required subsequently, Darcy's law (2.2b) and Laplace's equation (2.3) become

$$
\begin{gathered}
\mathbf{u}=\left(u_{r}, 0, u_{z}\right)=-k\left(\frac{\partial P}{\partial r}, 0, \frac{1}{\varepsilon^{2}} \frac{\partial P}{\partial z}\right), \\
\frac{1}{r} \frac{\partial}{\partial r}\left(r \frac{\partial P}{\partial r}\right)+\frac{1}{\varepsilon^{2}} \frac{\partial^{2} P}{\partial z^{2}}=0 .
\end{gathered}
$$

At leading order in the limit $\varepsilon \rightarrow 0$ the load condition (2.4) is simply

$$
L=2 \pi \int_{0}^{1} p r \mathrm{~d} r
$$

and the boundary conditions (2.5)-(2.8) and (2.11)-(2.13) are

$$
\begin{gathered}
v_{r}=0, \quad v_{z}=\frac{\mathrm{d} h}{\mathrm{~d} t} \quad \text { on } \quad z=h, \\
v_{z}=u_{z} \quad \text { on } \quad z=0, \\
P=p \quad \text { on } \quad z=0, \\
\frac{k^{1 / 2}}{\alpha} \frac{\partial v_{r}}{\partial z}=v_{r}-\delta u_{r} \quad \text { on } \quad z=0, \\
u_{z}=0 \quad \text { on } \quad z=-1,
\end{gathered}
$$




$$
\begin{gathered}
p=0 \quad \text { at } \quad r=1, \\
\frac{\partial p}{\partial r}=0 \quad \text { at } \quad r=0
\end{gathered}
$$

respectively. Note that a new dimensionless parameter $\delta$ has been introduced on the right-hand side of (2.27) so that it can be reduced to the non-dimensionalised version of (2.10), i.e. $v_{r}=0$ on $z=0$, by letting $\alpha \rightarrow \infty$ and setting $\delta=0$; otherwise $\delta=1$. The initial condition (2.14) becomes

$$
h(0)=d,
$$

where $d=H_{\mathrm{f}} / H_{\mathrm{p}}$ is the appropriately non-dimensionalised version of $H_{\mathrm{f}}$. For simplicity, in all of the plots presented subsequently we take $d=1$, corresponding to the initial thickness of the fluid layer being the same as the thickness of the porous bed.

\subsection{Unsteady Reynolds Equation}

Equation (2.20) shows immediately that, since the fluid layer is thin, the leading order fluid pressure is independent of $z$, i.e. $p=p(r, t)$. Solving the leading order version of (2.22), namely $\partial^{2} P / \partial z^{2}=0$, subject to the boundary conditions (2.26) and (2.28) using (2.21) shows that the leading order pore pressure is also independent of $z$, i.e. $P=P(r, t)$, and hence that at leading order (but not at higher order) the two pressures are the same, i.e. $P(r, t) \equiv p(r, t)$. The variation of $P$ with $z$ appears at first order in $\varepsilon^{2}$, and so seeking a solution to (2.22) in the form

$$
P=p(r, t)+\varepsilon^{2} P_{1}(r, z, t)+O\left(\varepsilon^{4}\right)
$$

and applying the no-penetration condition (2.28) yields

$$
\frac{\partial P_{1}}{\partial z}=-\frac{(1+z)}{r} \frac{\partial}{\partial r}\left(r \frac{\partial p}{\partial r}\right)
$$

Substituting (2.32) and (2.33) into (2.21) yields

$$
\mathbf{u}=\left(u_{r}, 0, u_{z}\right)=k\left(-\frac{\partial p}{\partial r}, 0, \frac{(1+z)}{r} \frac{\partial}{\partial r}\left(r \frac{\partial p}{\partial r}\right)\right) .
$$

Solving (2.16) and (2.19) for $v_{r}$ and $v_{z}$ subject to the boundary conditions (2.24a), (2.25) and (2.27) using (2.34) yields

$$
\begin{aligned}
& v_{r}=-\frac{(h-z)\left[\left(\alpha h+k^{1 / 2}\right) z+k^{1 / 2}\left(h+2 \delta \alpha k^{1 / 2}\right)\right]}{2\left(\alpha h+k^{1 / 2}\right)} \frac{\partial p}{\partial r} \\
& v_{z}=\frac{2\left(\alpha h+k^{1 / 2}\right)\left(6 k-z^{3}\right)+3 \alpha\left(h^{2}-2 \delta k\right) z^{2}+6 k^{1 / 2} h\left(h+2 \delta \alpha k^{1 / 2}\right) z}{12 r\left(\alpha h+k^{1 / 2}\right)} \frac{\partial}{\partial r}\left(r \frac{\partial p}{\partial r}\right) .
\end{aligned}
$$

Substituting (2.36) into (2.24b) we obtain the unsteady Reynolds equation

$$
\frac{1}{r} \frac{\partial}{\partial r}\left(r \frac{\partial p}{\partial r}\right)=\frac{12\left(\alpha h+k^{1 / 2}\right)}{h^{2}\left(\alpha h^{2}+4 k^{1 / 2} h+6 \delta \alpha k\right)+12 k\left(\alpha h+k^{1 / 2}\right)} \frac{\mathrm{d} h}{\mathrm{~d} t},
$$

which, subject to the load condition (2.23), the pressure boundary conditions (2.29) and (2.30), and the initial condition (2.31), determines the pressure $p=p(r, t)$ and the fluid layer thickness $h=h(t)$. This system will be solved subsequently in $\S 3$. 


\subsection{Streamfunction}

The streamfunction $\psi=\psi(r, z, t)$ (non-dimensionalised with $V \mathscr{R} H_{\mathrm{p}}=L_{0} H_{\mathrm{p}}^{3} /\left(\mu \mathscr{R}^{2}\right)$ ) satisfies

$$
v_{r}=-\frac{1}{r} \frac{\partial \psi}{\partial z}, \quad v_{z}=\frac{1}{r} \frac{\partial \psi}{\partial r}
$$

in the fluid layer $0 \leqslant z \leqslant h$, and

$$
u_{r}=-\frac{1}{r} \frac{\partial \psi}{\partial z}, \quad u_{z}=\frac{1}{r} \frac{\partial \psi}{\partial r}
$$

in the porous bed $-1 \leqslant z \leqslant 0$, and the boundary conditions

$$
\psi=0 \quad \text { on } \quad z=-1, \quad \lim _{z \rightarrow 0^{+}} \psi=\lim _{z \rightarrow 0^{-}} \psi .
$$

Solving (2.38) and (2.39) for $\psi$ subject to the boundary conditions (2.40) yields

$$
\psi=\frac{r\left[2\left(\alpha h+k^{1 / 2}\right)\left(6 k-z^{3}\right)+3 \alpha\left(h^{2}-2 \delta k\right) z^{2}+6 k^{1 / 2} h\left(h+2 \delta \alpha k^{1 / 2}\right) z\right]}{12\left(\alpha h+k^{1 / 2}\right)} \frac{\partial p}{\partial r}
$$

in the fluid layer $0 \leqslant z \leqslant h$, and

in the porous bed $-1 \leqslant z \leqslant 0$.

$$
\psi=k r(1+z) \frac{\partial p}{\partial r}
$$

\subsection{Fluid Particle Paths}

The path $(r, z)=(r(t), z(t))$ taken by a fluid particle, initially situated at the point $\left(r_{0}, z_{0}\right)$ (where $0 \leqslant$ $r_{0} \leqslant 1$ and $\left.-1 \leqslant z_{0} \leqslant d\right)$ is governed by the non-autonomous ordinary differential equations

$$
\frac{\mathrm{d} r}{\mathrm{~d} t}=v_{r}, \quad \frac{\mathrm{d} z}{\mathrm{~d} t}=v_{z}
$$

in the fluid layer $0 \leqslant z \leqslant h$, and

$$
\frac{\mathrm{d} r}{\mathrm{~d} t}=\frac{u_{r}}{\phi}, \quad \frac{\mathrm{d} z}{\mathrm{~d} t}=\frac{u_{z}}{\phi}
$$

in the porous bed $-1 \leqslant z \leqslant 0$.

\section{General Solution}

\subsection{Pressure and Velocities}

Integrating the unsteady Reynolds equation (2.37) twice with respect to $r$ and applying the pressure boundary conditions (2.29) and (2.30) we obtain

$$
p=-\frac{3\left(\alpha h+k^{1 / 2}\right)\left(1-r^{2}\right)}{h^{2}\left(\alpha h^{2}+4 k^{1 / 2} h+6 \delta \alpha k\right)+12 k\left(\alpha h+k^{1 / 2}\right)} \frac{\mathrm{d} h}{\mathrm{~d} t} .
$$

Applying the load condition (2.23) yields a separable first-order ordinary differential equation for the fluid layer thickness $h=h(t)$ :

$$
L(t)=-\frac{3 \pi\left(\alpha h+k^{1 / 2}\right)}{2\left[h^{2}\left(\alpha h^{2}+4 k^{1 / 2} h+6 \delta \alpha k\right)+12 k\left(\alpha h+k^{1 / 2}\right)\right]} \frac{\mathrm{d} h}{\mathrm{~d} t} .
$$


Re-arranging (3.2) for $\mathrm{d} h / \mathrm{d} t$ and substituting into (3.1) reveals that the pressure is simply

$$
p=\frac{2\left(1-r^{2}\right) L(t)}{\pi},
$$

showing that the pressure is parabolic in $r$ and independent of $k$, and that its only temporal dependence is via that of the load $L(t)$. Substituting (3.3) into (2.34) shows that the Darcy velocity is given by

$$
\mathbf{u}=\left(u_{r}, 0, u_{z}\right)=\frac{4 L(t) k}{\pi}(r, 0,-2(1+z)),
$$

which is independent of both $\alpha$ and $\delta$, and so is the same for all three choices of the boundary conditions (2.8)-(2.10). Substituting (3.3) into (2.35) and (2.36) shows that the fluid velocity is given by $\mathbf{v}=$ $\left(v_{r}, 0, v_{z}\right)$, where

$$
\begin{aligned}
& v_{r}=\frac{2 L(t)(h-z)\left[\left(\alpha h+k^{1 / 2}\right) z+k^{1 / 2}\left(h+2 \delta \alpha k^{1 / 2}\right)\right] r}{\pi\left(\alpha h+k^{1 / 2}\right)}, \\
& v_{z}=-\frac{2 L(t)\left[2\left(\alpha h+k^{1 / 2}\right)\left(6 k-z^{3}\right)+3 \alpha\left(h^{2}-2 \delta k\right) z^{2}+6 k^{1 / 2} h\left(h+2 \delta \alpha k^{1 / 2}\right) z\right]}{3 \pi\left(\alpha h+k^{1 / 2}\right)} .
\end{aligned}
$$

In particular, (3.4)-(3.6) show that both $u_{r}$ and $v_{r}$ are linear in $r$, but that both $u_{z}$ and $v_{z}$ are independent of $r$.

\subsection{Fluid Layer Thickness}

Integrating (3.2) from 0 to $t$ and imposing the initial condition (2.31) yields an implicit solution for the fluid layer thickness $h=h(t)$ :

$$
\int_{0}^{t} L(\tau) \mathrm{d} \tau=\frac{3 \pi}{2} \int_{h}^{d} \frac{\alpha s+k^{1 / 2}}{s^{2}\left(\alpha s^{2}+4 k^{1 / 2} s+6 \delta \alpha k\right)+12 k\left(\alpha s+k^{1 / 2}\right)} \mathrm{d} s .
$$

For simplicity in the remainder of the present work we concentrate on the case of constant (dimensional) load $L=L_{0}>0$, corresponding to taking the constant (dimensionless) load to be equal to unity, i.e. $L_{0}=1$, without loss of generality. We shall, however, retain the constant (dimensionless) load $L_{0}$ explicitly in what follows for clarity of presentation.

In the case of constant load $L=L_{0}$ equation (3.7) yields an implicit expression for the fluid layer thickness $h=h(t)$ at time $t$ in the form of an explicit expression for the time $t=t(h)$ taken for the fluid layer to reduce to a thickness $h$ :

$$
t(h)=\frac{3 \pi}{2 L_{0}} \int_{h}^{d} \frac{\alpha s+k^{1 / 2}}{s^{2}\left(\alpha s^{2}+4 k^{1 / 2} s+6 \delta \alpha k\right)+12 k\left(\alpha s+k^{1 / 2}\right)} \mathrm{d} s .
$$

In the special case in which the bed is impermeable, i.e. when $k=0$, the fluid layer thickness $h=h(t)$ is given explicitly by

$$
h=\left(\frac{3 \pi d^{2}}{3 \pi+4 d^{2} L_{0} t}\right)^{1 / 2}
$$

so that

$$
h \sim\left(\frac{3 \pi}{4 L_{0} t}\right)^{1 / 2} \rightarrow 0^{+} \quad \text { as } t \rightarrow \infty
$$


(a)

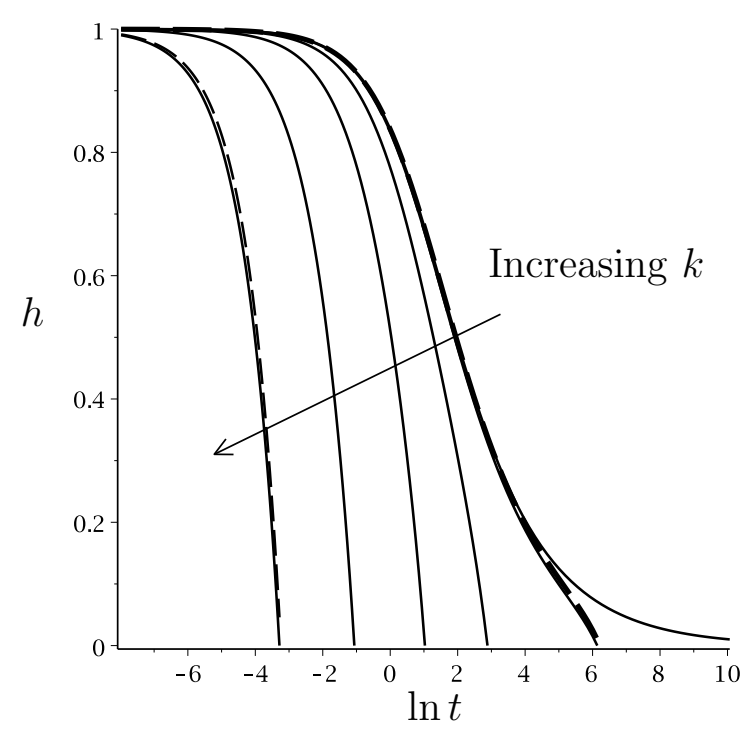

(b)

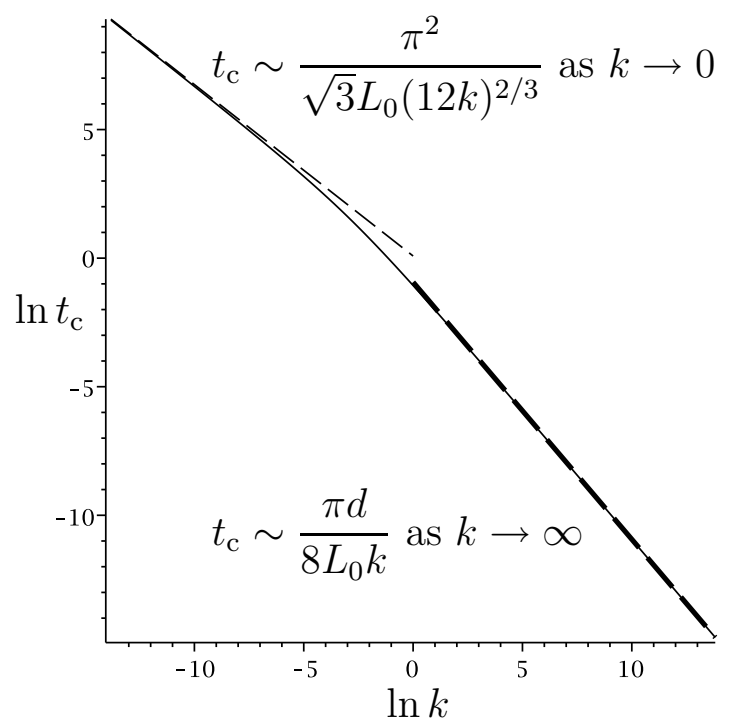

FIG. 2. (a) Plots of the fluid layer thickness $h$ as a function of $\ln t$ given by (3.8) for $k=0,10^{-4}, 10^{-2}, 10^{-1}, 1$ and 10 (solid lines), the uniformly valid leading order small- $k$ asymptotic solution (4.33) for $k=10^{-4}$ (dashed line) and the leading order large- $k$ asymptotic solution (B.7) for $k=10$ (bold dashed line). (b) Plots of $\ln t_{\mathrm{c}}$ as a function of $\ln k$ given by (3.11) (solid line), the leading order small $-k$ asymptotic solution (4.32) (dashed line) and the leading order large- $k$ asymptotic solution (B.9) (bold dashed line). In all of the plots $\alpha=1$.

i.e. an infinite time is required to squeeze all of the fluid out of the fluid layer (see, for example, Stone (2005)).

In the general case $k \neq 0$ the integral in (3.8) was evaluated numerically using the int tool (with its default numerical method) in the computer algebra and numerical analysis package MAPLE. (Note that the integral in (3.8) may, in principle, be evaluated analytically, though this involves the zeros of the quartic expression appearing in the denominator of the integrand. These zeros may, in principle, be found using standard formulae; however, in practice, they are very unwieldy and so we always evaluated the integral numerically.)

Figure 2(a) shows plots of $h$ as a function of $\ln t$ for different values of the permeability $k$. Figure 2(a) shows that, as expected, increasing $k$ decreases the time taken for the fluid layer to reduce to a thickness $h$. Figure 2(a) also shows that when the bed is permeable, i.e. when $k \neq 0$, the bearing always makes contact with the porous bed in a finite time. This finite contact time is denoted by $t_{\mathrm{c}}$, and is given by setting $h=0$ in (3.8) to yield

$$
t_{\mathrm{c}}=\frac{3 \pi}{2 L_{0}} \int_{0}^{d} \frac{\alpha s+k^{1 / 2}}{s^{2}\left(\alpha s^{2}+4 k^{1 / 2} s+6 \delta \alpha k\right)+12 k\left(\alpha s+k^{1 / 2}\right)} \mathrm{d} s .
$$

Figure 2(b) shows a plot of $\ln t_{\mathrm{c}}$ as a function of $\ln k$. Figure 2(b) shows that, in agreement with Figure 2 (a), increasing $k$ decreases the contact time $t_{\mathrm{c}}$. (In fact, it will be shown in $\S 4.1$ that $t_{\mathrm{c}}=O\left(k^{-2 / 3}\right) \gg 1$ in the limit $k \rightarrow 0$ and in Appendix B that $t_{\mathrm{c}}=O\left(k^{-1}\right) \ll 1$ in the limit $k \rightarrow \infty$.) 
10 of 35

(a)

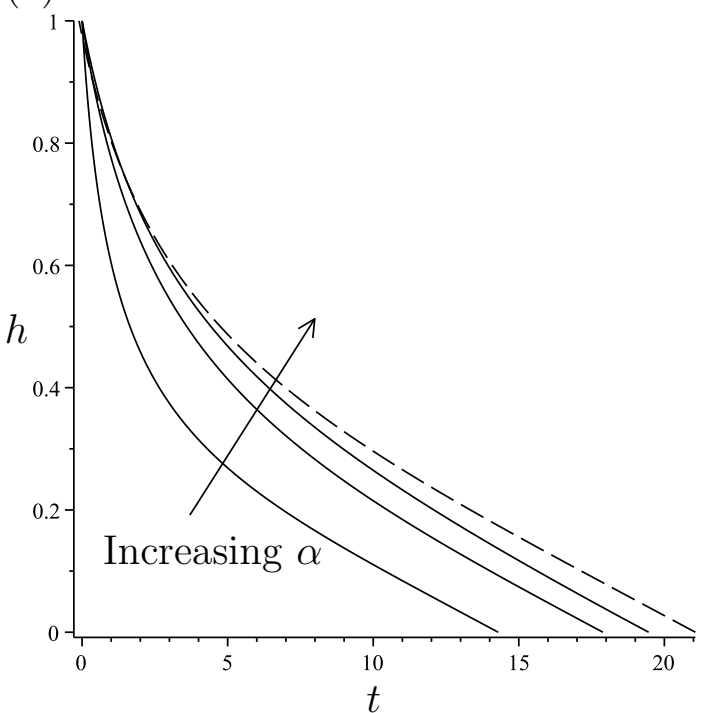

D. J. Knox et al.

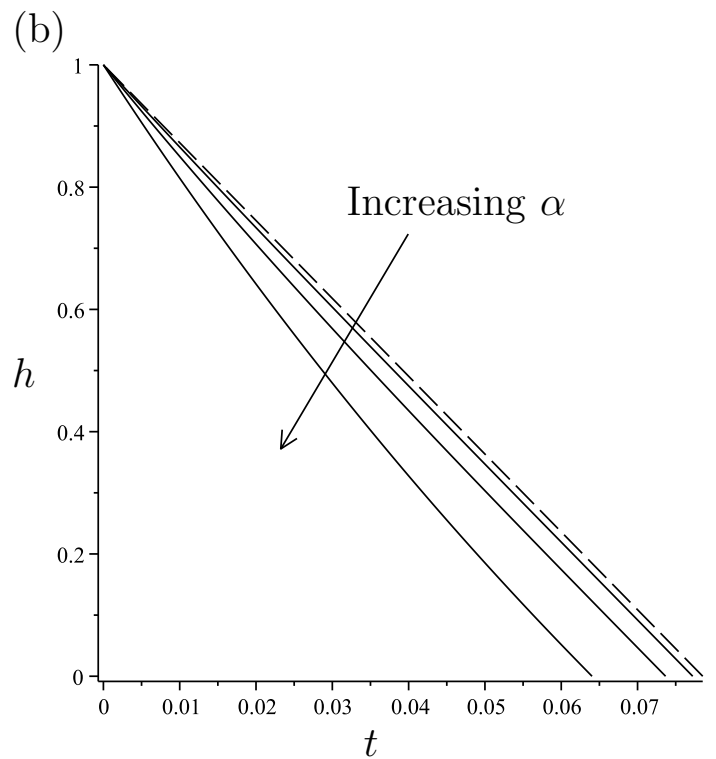

FIG. 3. (a) Plots of the fluid layer thickness $h$ as a function of $t$ given by (3.8) for $\alpha=10^{-2}, \alpha=1$ and $\alpha=100$ with $k=10^{-2}$ (solid lines) and the uniformly valid leading order small- $k$ asymptotic solution (4.33) with $k=10^{-2}$ (dashed line). (b) Plots of $h$ as a function of $t$ given by (3.8) for $\alpha=10^{-2}, \alpha=1$ and $\alpha=100$ with $k=5$ (solid lines) and the leading order large- $k$ asymptotic solution (B.7) with $k=5$ (dashed line).

While the effect of varying the permeability $k$ is relatively straightforward to understand, the effect of varying the Beavers-Joseph constant $\alpha$ is more subtle. Figure 3 shows plots of $h$ as a function of $t$ for different values of $\alpha$ for two values of $k$. In particular, Figure 3(a) shows that for a "small" value of $k$ (specifically $k=10^{-2}$ ) as $\alpha$ increases the time taken for the fluid layer to reduce to a thickness $h$ increases, whereas Figure 3(b) shows that for a "large" value of $k$ (specifically $k=5$ ) as $\alpha$ increases the time taken for the fluid layer to reduce to a thickness $h$ decreases. This qualitative change in behaviour is confirmed by the analysis of the asymptotic limits $k \rightarrow 0$ and $k \rightarrow \infty$ given subsequently in $\S 4$ and Appendix B, respectively.

To understand the physical reason why varying the Beavers-Joseph constant $\alpha$ has a qualitatively different effect for small and large values of the permeability $k$ it is instructive to examine the radial fluid velocity $v_{r}$ and the radial Darcy velocity $u_{r}$. Figure 4 shows plots of $v_{r}$ as a function of $z$ for $0 \leqslant z \leqslant h$ and of $u_{r}$ as a function of $z$ for $-1 \leqslant z \leqslant 0$ for different values of $\alpha$ for three values of the permeability $k$. (Note that all of the plots shown in Figure 4, and also all of those shown subsequently in Figure 5, are for the same value of the fluid layer thickness, namely $h=1 / 2$, and so are therefore not at the same instant $t$.) Figure 4(a) shows the flow in the special case in which the bed is impermeable, i.e. when $k=0$ and hence $l_{\mathrm{s}}=0$, in which case there is, of course, no flow in the porous bed and no slip between the fluid and the porous bed at the interface $z=0$. Figures 4(b) and 4(c) show the flow for different values of $\alpha$ for a "small" (specifically $k=10^{-2}$ ) and a "large" (specifically $k=10$ ) value of the permeability $k$, respectively. Note that, since $u_{r}$ is independent of both $\alpha$ and $z$, there is only a single uniform velocity 
(a)

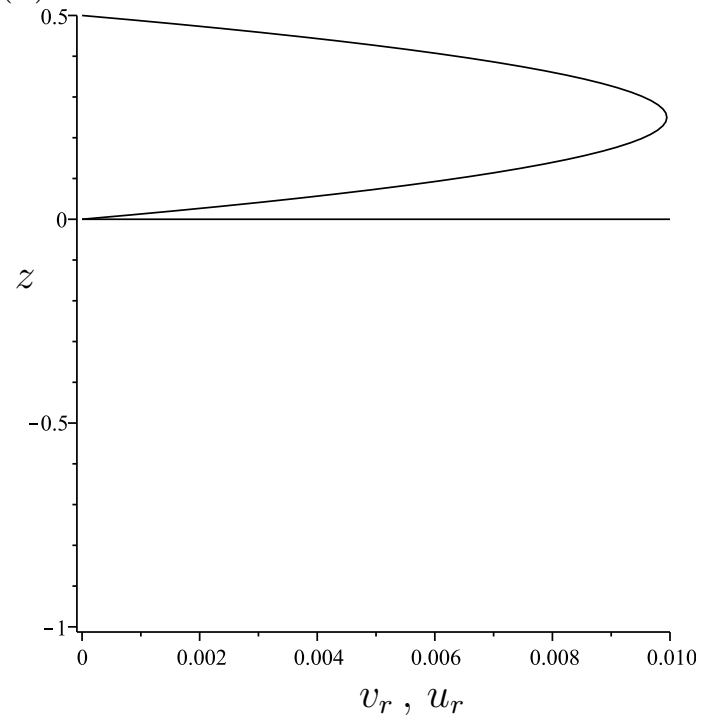

(b)

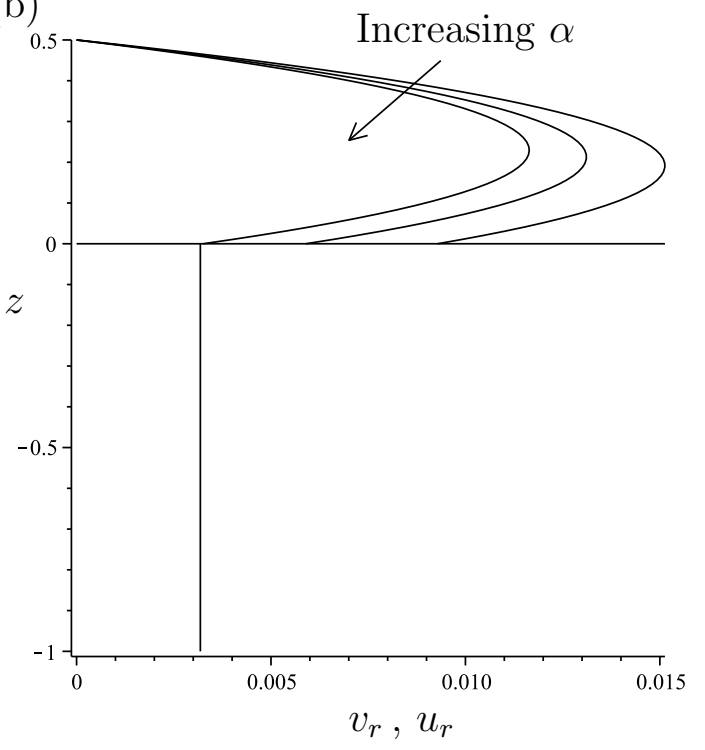

(c)

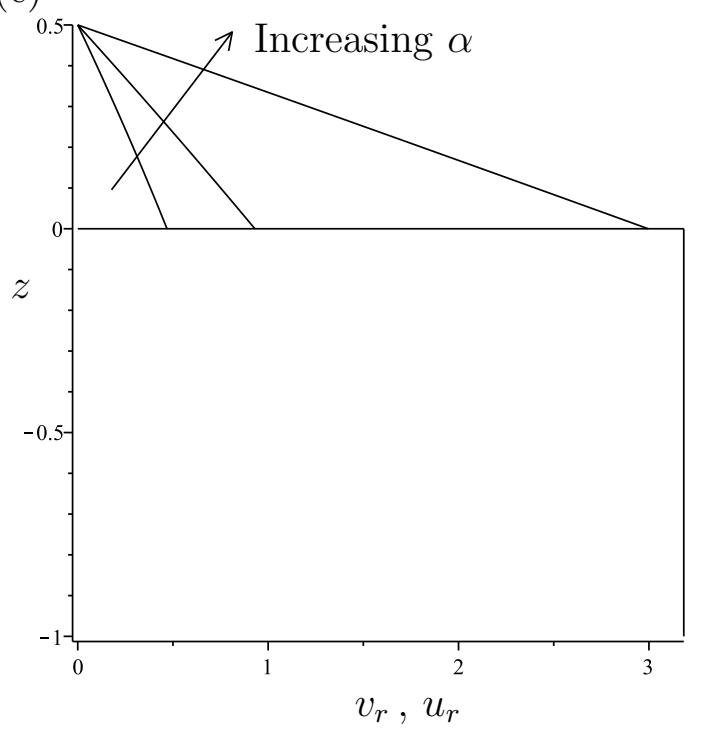

FIG. 4. Plots of the radial fluid velocity $v_{r}$ as a function of $z$ for $0 \leqslant z \leqslant h$ and of the radial Darcy velocity $u_{r}$ as a function of $z$ for $-1 \leqslant z \leqslant 0$ at $r=1 / 4$ when $h=1 / 2$ for $\alpha=1,2.5$ and 100 with (a) $k=0$, (b) $k=10^{-2}$ and (c) $k=10$. 
profile in the porous bed in Figures 4(b) and 4(c). These figures also clearly show the effect of velocity slip at the interface $z=0$, and that the fastest flow is in the fluid layer for small $k$ but in the porous bed for large $k$. In particular, Figure 4(b) shows that when $k$ is small increasing $\alpha$ (i.e. decreasing the slip length $l_{\mathrm{s}}$ ) decreases $v_{r}$ and hence decreases the fluid volume flux out of the fluid layer into the region beyond $r=1$, denoted by $Q_{\text {out }}$ and given by

$$
Q_{\text {out }}=\left.2 \pi \int_{0}^{h} v_{r} r\right|_{r=1} \mathrm{~d} z=\frac{2 L_{0} h^{2}\left(\alpha h^{2}+4 k^{1 / 2} h+6 \delta \alpha k\right)}{3\left(\alpha h+k^{1 / 2}\right)} .
$$

Since the fluid volume flux into the porous bed, denoted by $Q_{\text {bed }}$ and given by

$$
Q_{\text {bed }}=\left.2 \pi \int_{0}^{1} v_{z} r\right|_{z=0} \mathrm{~d} r=-8 L_{0} k
$$

is constant and independent of $\alpha$, this decrease in $Q_{\text {out }}$ causes the time taken for the fluid layer to reduce to a thickness $h$ to increase, consistent with the behaviour for small $k$ shown in Figure 3(a). On the other hand, Figure 4(c) shows that when $k$ is large increasing $\alpha$ (i.e. decreasing the slip length $l_{\mathrm{s}}$ ) increases $v_{r}$ and hence increases $Q_{\text {out }}$, causing the time taken for the fluid layer to reduce to a thickness $h$ to decrease, consistent with the behaviour for large $k$ shown in Figure 3(b).

\subsection{Instantaneous Streamlines}

Figure 5 shows plots of the instantaneous streamlines for three values of the permeability $k$. Figure 5(a) shows the streamlines in the special case in which the bed is impermeable, i.e. when $k=0$, in which case there is, of course, no flow in the porous bed. Figures 5(b) and 5(c) show the streamlines for two non-zero values of the permeability $k$. In particular, they show that when the bed is permeable the vertical fluid velocity $v_{z}$ on the interface $z=0$ (which is given by $\left.v_{z}\right|_{z=0}=\left.u_{z}\right|_{z=0}=-8 L_{0} k / \pi$ ) is always negative, and hence that fluid always flows from the fluid layer into the porous bed and never flows the other way. They also show that as $k$ increases so do $Q_{\text {out }}$ and $Q_{\text {bed }}$, and hence that the contact time $t_{\mathrm{c}}$ decreases, consistent with the behaviour shown in Figure 2.

\subsection{Fluid Particle Paths and Penetration Depths}

Using (3.2) to eliminate $t$ from (2.43) and (2.44), the differential equations that govern the path $(r, z)$ taken by a fluid particle are

$$
\begin{aligned}
\frac{1}{r} \frac{\mathrm{d} r}{\mathrm{~d} h} & =-\frac{3(h-z)\left[\left(\alpha h+k^{1 / 2}\right) z+k^{1 / 2}\left(h+2 \delta \alpha k^{1 / 2}\right)\right]}{h^{2}\left(\alpha h^{2}+4 k^{1 / 2} h+6 \delta \alpha k\right)+12 k\left(\alpha h+k^{1 / 2}\right)} \\
\frac{\mathrm{d} z}{\mathrm{~d} h} & =\frac{2\left(\alpha h+k^{1 / 2}\right)\left(6 k-z^{3}\right)+3 \alpha\left(h^{2}-2 \delta k\right) z^{2}+6 k^{1 / 2} h\left(h+2 \delta \alpha k^{1 / 2}\right) z}{h^{2}\left(\alpha h^{2}+4 k^{1 / 2} h+6 \delta \alpha k\right)+12 k\left(\alpha h+k^{1 / 2}\right)}
\end{aligned}
$$

in the fluid layer $0 \leqslant z \leqslant h$, and

$$
\begin{aligned}
\frac{1}{r} \frac{\mathrm{d} r}{\mathrm{~d} h} & =-\frac{6 k\left(\alpha h+k^{1 / 2}\right)}{\phi\left[h^{2}\left(\alpha h^{2}+4 k^{1 / 2} h+6 \delta \alpha k\right)+12 k\left(\alpha h+k^{1 / 2}\right)\right]}, \\
\frac{1}{1+z} \frac{\mathrm{d} z}{\mathrm{~d} h} & =\frac{12 k\left(\alpha h+k^{1 / 2}\right)}{\phi\left[h^{2}\left(\alpha h^{2}+4 k^{1 / 2} h+6 \delta \alpha k\right)+12 k\left(\alpha h+k^{1 / 2}\right)\right]}
\end{aligned}
$$


(a)

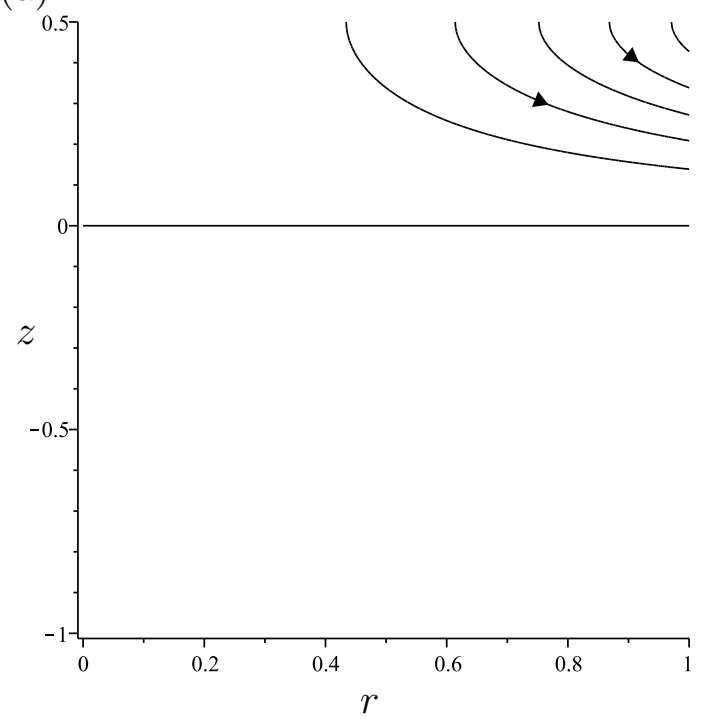

(b)

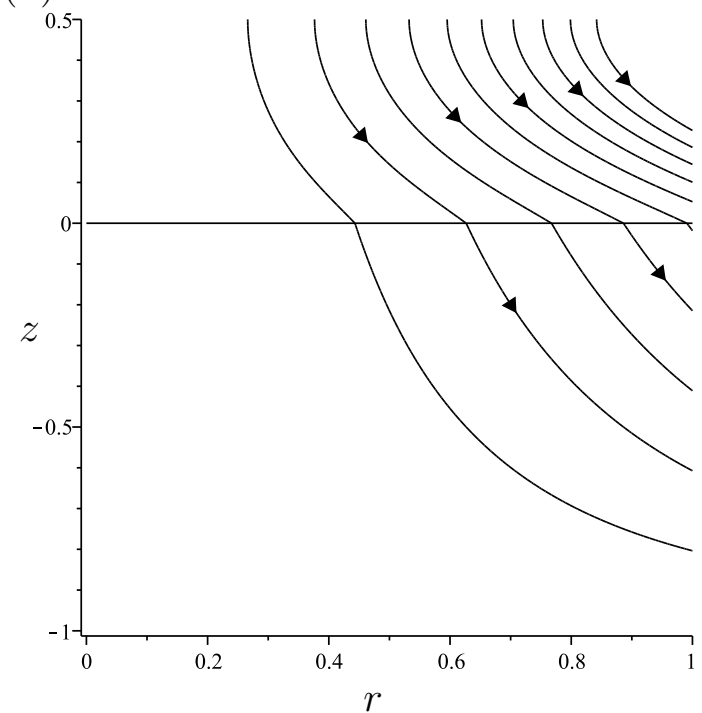

(c)

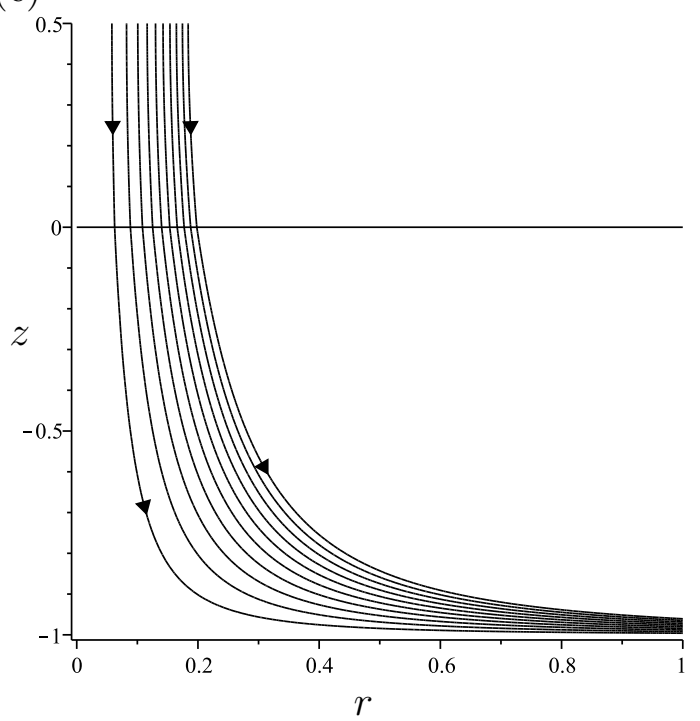

FIG. 5. Plots of the instantaneous streamlines $\psi=$ constant $=-j / 4 \times 10^{-2}$ for $j=1,2, \ldots, 10$ with $\psi$ given by (2.41) for $0 \leqslant z \leqslant h$ and (2.42) for $-1 \leqslant z<0$ when $h=1 / 2$ for (a) $k=0$, (b) $k=10^{-2}$ and (c) $k=1 / 2$. In all of the plots $\alpha=1$. 
in the porous bed $-1 \leqslant z \leqslant 0$, subject to the initial conditions

$$
r=r_{0} \quad \text { and } \quad z=z_{0} \quad \text { when } \quad t=0 \text {, i.e. when } h=d .
$$

Integrating (3.16) and (3.17) subject to (3.18) shows that the particle paths in the porous bed are given by

$$
\left(\frac{r}{r_{0}}\right)^{2}=\frac{1+z_{0}}{1+z}=\exp \left[-\frac{12 k}{\phi} \int_{d}^{h} \frac{\alpha s+k^{1 / 2}}{s^{2}\left(\alpha s^{2}+4 k^{1 / 2} s+6 \delta \alpha k\right)+12 k\left(\alpha s+k^{1 / 2}\right)} \mathrm{d} s\right],
$$

and so, in particular, always satisfy $r^{2}(1+z)=r_{0}^{2}\left(1+z_{0}\right)=$ constant.

Figure 6 shows the paths $(r, z)$ taken by several fluid particles from their initial positions $\left(r_{0}, z_{0}\right)$ at $t=0$ for three values of the permeability $k$.

In the special case in which the bed is impermeable, i.e. when $k=0$, equations (3.14) and (3.15) may be solved exactly subject to (3.18) to yield

$$
\begin{aligned}
r & =r_{0}\left(\frac{4 d\left(d-z_{0}\right) z_{0}+\left(d-2 z_{0}\right)^{2} h}{d^{2} h}\right)^{3 / 4}, \\
z & =\frac{h}{2}\left[1-\left(d-2 z_{0}\right)\left(\frac{h}{4 d\left(d-z_{0}\right) z_{0}+\left(d-2 z_{0}\right)^{2} h}\right)^{1 / 2}\right]
\end{aligned}
$$

for $z_{0} \geqslant 0$. Figure 6 (a) shows the paths of several fluid particles in this case and, in particular, shows how all of the fluid particles eventually flow out of the fluid layer into the region beyond $r=1$.

In the general case $k \neq 0$ the differential equations (3.14)-(3.17) were solved numerically using the dsolve tool (with its default numerical method) in MAPLE subject to (3.18). Figures 6(b) and 6(c) show the paths of several fluid particles for two non-zero values of the permeability $k$. In particular, they show that fluid particles initially in the fluid layer can either flow out of the fluid layer into the region beyond $r=1$ or flow into the porous bed and then, in some cases, out of the porous bed into the region beyond $r=1$. Since the vertical Darcy velocity $u_{z}=-8 L_{0} k(1+z) / \pi$ is less than or equal to zero, fluid particles that enter the porous bed never re-enter the fluid layer, and fluid particles that are initially in the porous bed never enter the fluid layer. Figures 6(b) and 6(c) also show that as $k$ increases then $v_{z}$ increases relative to $v_{r}$ so that the flow in the fluid layer is increasingly directed towards the porous bed, and consequently the fraction of the fluid particles initially in the fluid layer that flow into the porous bed increases.

Fluid particles initially in the fluid layer that enter the porous bed do so by crossing the interface $z=0$ at some point $\left(r^{*}, 0\right)$, where $r^{*}=r^{*}\left(r_{0}, z_{0}\right)$, at $t=t^{*}$ corresponding to $h=h\left(t^{*}\right)=h^{*}$, and so the critical curve that divides the region of particles that enter the porous bed (with $r^{*}<1$ ) from the region of those that do not is given by $r^{*}=1$. The curve $r^{*}=1$ is found by solving (3.14) and (3.15) subject to $r=1$ and $z=0$ at $h=h^{*}$ backwards from $h=h^{*}$ to $h=d$. Evaluating the solution for $(r, z)$ when $h=d$ yields the initial position $\left(r_{0}, z_{0}\right)$ of the fluid particle that passes through the point $(r, z)=(1,0)$ when $h=h^{*}$. Repeating this procedure for $0 \leqslant h^{*} \leqslant d$ yields the curve $r^{*}=1$. Figures $6(\mathrm{~b})$ and $6(\mathrm{c})$ include plots of the curve $r^{*}=1$ calculated in this way and confirm that fluid particles that initially lie to the left of $r^{*}=1$ flow from the fluid layer into the porous bed, and the fluid particles that initially lie to the right of $r^{*}=1$ flow out of the fluid layer into the region beyond $r=1$. Figure 7 shows plots of the curve $r^{*}=1$ for different values of the permeability $k$. In particular, Figure 7 shows that as $k$ increases the 

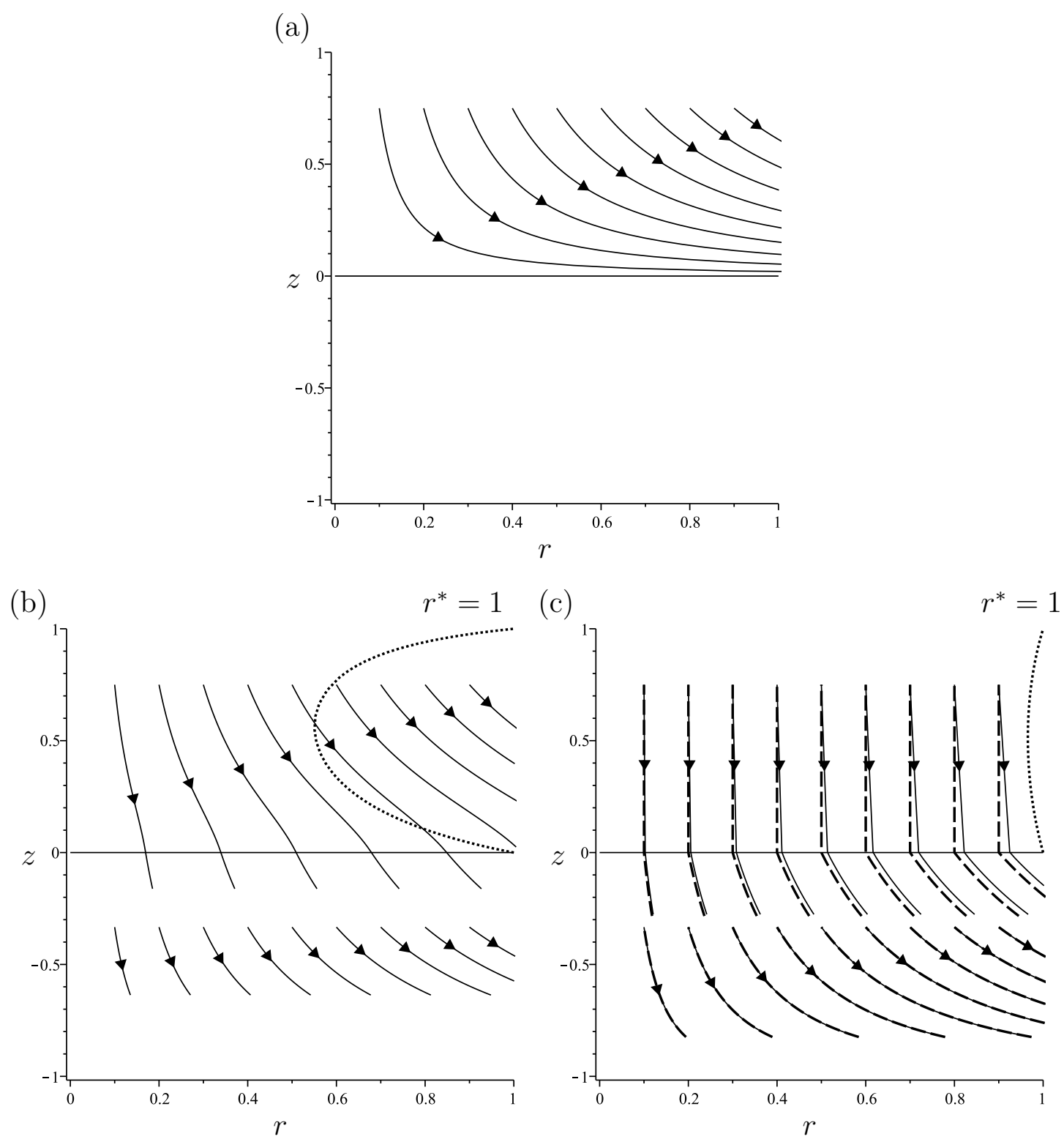

FIG. 6. Plots of fluid particle paths $(r, z)$ with $r$ and $z$ given by (a) (3.20) and (3.21) for $\left(r_{0}, z_{0}\right)=(0.1,0.75),(0.2,0.75), \ldots$, $(0.9,0.75),(\mathrm{b}, \mathrm{c})(3.14)-(3.17)$ subject to $\left(r_{0}, z_{0}\right)=(0.1,0.75),(0.2,0.75), \ldots,(0.9,0.75)$ and $\left(r_{0}, z_{0}\right)=(0.1,-1 / 3),(0.2,-1 / 3)$, $\ldots,(0.9,-1 / 3)$ for (b) $k=10^{-2}$ and (c) $k=10$ (solid lines) and the leading order large- $k$ asymptotic solutions (B.12), (B.16), (B.17), (B.19) and (B.20) (dashed lines) for $\alpha=1$ and $\phi=3 / 4$. Parts (b) and (c) also include plots of the curve $r^{*}=1$ (dotted lines). 


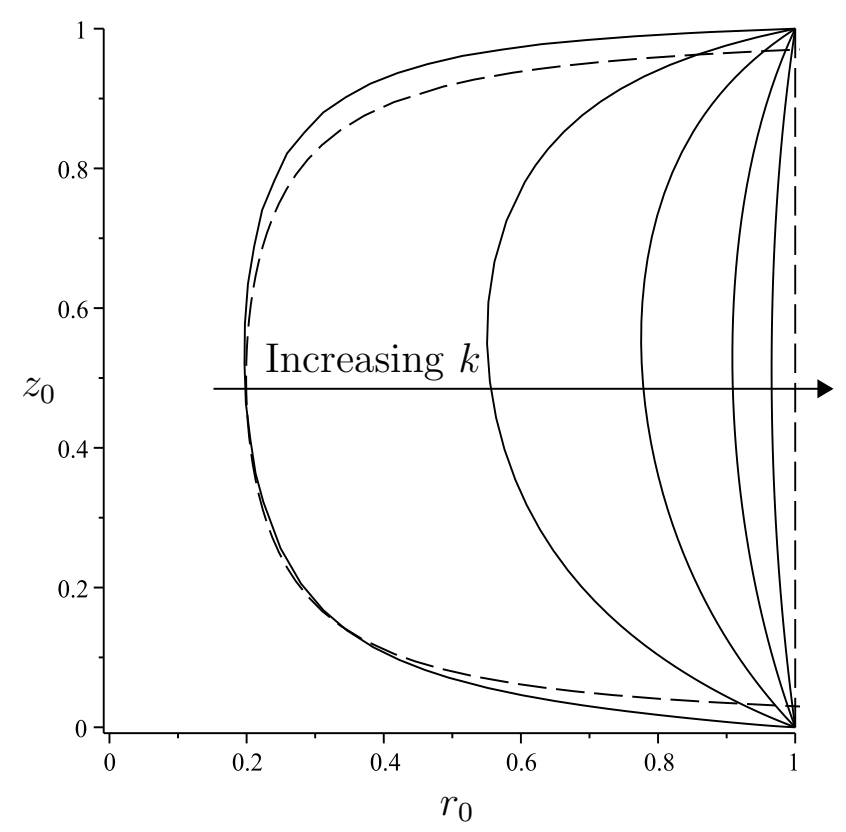

FIG. 7. Plots of the critical curve $r^{*}=1$ which divides the region of particles that enter the porous bed $\left(\right.$ with $\left.r^{*}<1\right)$ from those that do not (with $r^{*}>1$ ) for $k=10^{-4}, 10^{-2}, 10^{-1}, 1$ and 10 (solid lines), the leading order small- $k$ asymptotic solution (4.44) for $k=10^{-4}$ (dashed line) and the leading order large- $k$ asymptotic solution $r_{0}=1$ (dashed line). In all of the plots $\alpha=1$.

curve $r^{*}=1$ moves to the right, i.e. less fluid flows out of the fluid layer into the region beyond $r=1$ and more fluid flows into the porous bed.

Also of considerable practical interest are the depths to which fluid particles (which may be initially situated in either the fluid layer or the porous bed) penetrate into the porous bed, denoted by $z_{\text {pen }}$ and given by $z_{\text {pen }}=z\left(t_{\mathrm{c}}\right)<0$. Since both $v_{z}$ and $u_{z}$ are independent of $r, z_{\text {pen }}$ is independent of $r_{0}$. However, the value of $r_{0}$ does affect whether or not a fluid particle flows into the region beyond $r=1$. Figure 8(a) shows plots of $\ln \left|z_{\text {pen }}\right|$ as a function of $\ln k$ for "small" values of the permeability $k$ for three fluid particles initially situated in the fluid layer with $r_{0}=O\left(k^{1 / 4}\right)$. In particular, Figure 8(a) shows that $\left|z_{\text {pen }}\right|$ decreases as $k$ decreases and/or $z_{0}$ increases. Moreover, the effect of $z_{0}$ on $z_{\text {pen }}$ becomes less pronounced as $k$ decreases. (In fact, it will be shown in $\S 4.2$ that at leading order in the limit of small permeability $k \rightarrow 0$ the penetration depth $z_{\text {pen }}=O\left(k^{1 / 3}\right) \ll 1$ of a fluid particle initially situated in the fluid layer with $r_{0}=O\left(k^{1 / 4}\right)$ is small and independent of $z_{0}$.) Figure 8(b) shows plots of $z_{\text {pen }}$ as a function of $\ln k$ for "large" values of the permeability $k$ for three fluid particles initially situated in the fluid layer. In particular, Figure 8(b) shows that again $\left|z_{\text {pen }}\right|$ decreases as $k$ decreases and/or $z_{0}$ increases. However, in this case, the effect of $k$ on $z_{\text {pen }}$ becomes less pronounced as $k$ increases. (In fact, it will be shown in Appendix B that at leading order in the limit $k \rightarrow \infty$ the penetration depth $z_{\text {pen }}=O(1)$ of a fluid particle initially situated in the fluid layer is independent of $k$ and its magnitude decreases monotonically as $z_{0}$ increases.) Figure 8(c) shows plots of $\ln \left|z_{\text {pen }}-z_{0}\right|$ as a function of $\ln k$ for "small" values of the permeability $k$ for three fluid particles initially situated in the porous bed. In particular, Figure 8(c) shows that $\left|z_{\text {pen }}-z_{0}\right|$ decreases as $k$ decreases and/or $z_{0}$ decreases. (In fact, it will be shown in $\S 4.2$ that at leading order in the limit $k \rightarrow 0$ the magnitude of the relative penetration depth 
(a)

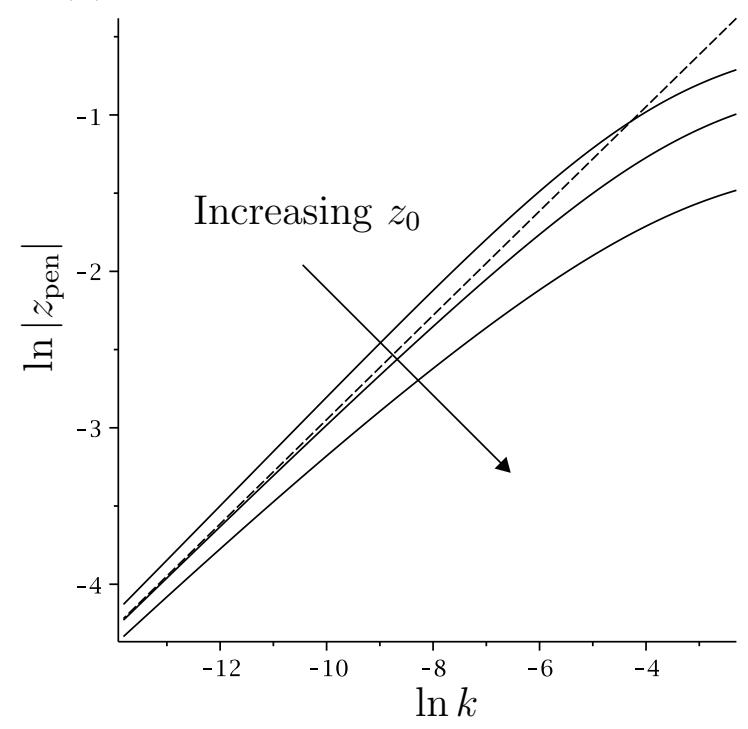

(c)

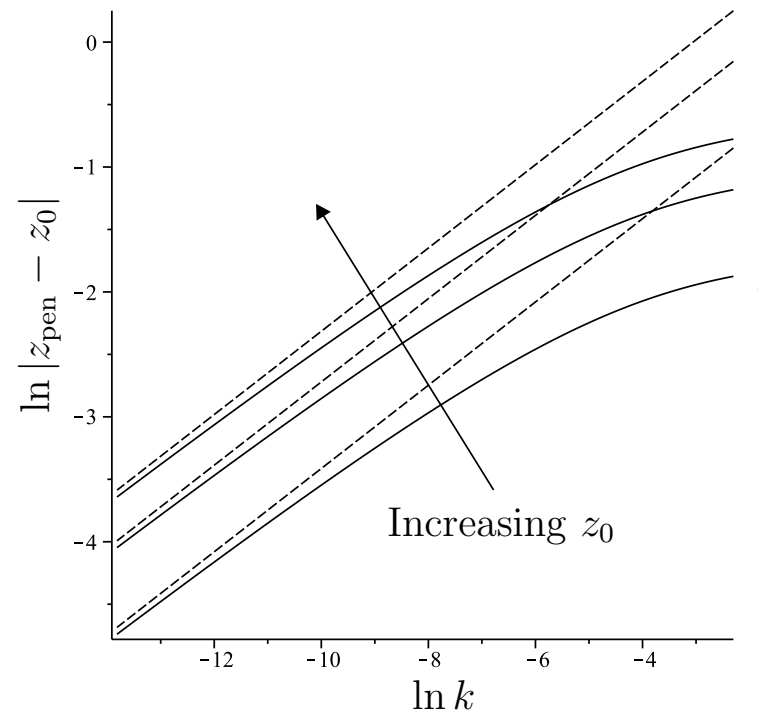

(b)

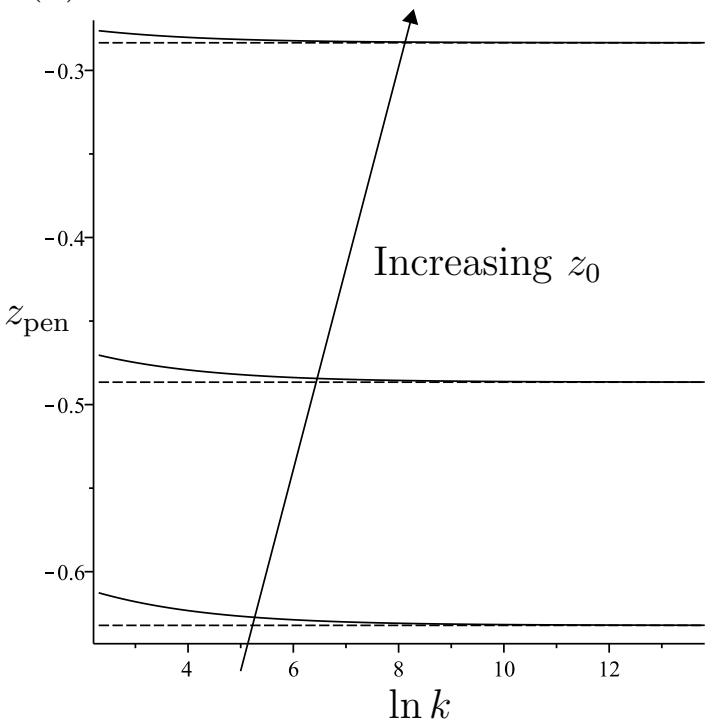

(d)

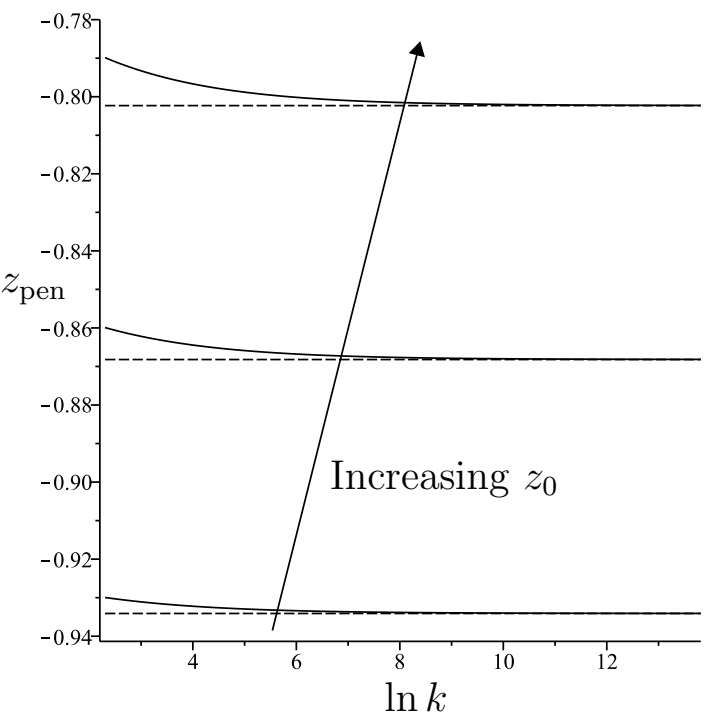

FIG. 8. (a) Plots of $\ln \left|z_{\text {pen }}\right|$ as a function of $\ln k$ given by (3.14)-(3.17) subject to $\left(r_{0}, z_{0}\right)=\left(0.5 \times(12 k)^{1 / 4}, 0.25\right),(0.5 \times$ $\left.(12 k)^{1 / 4}, 0.5\right)$ and $\left(0.5 \times(12 k)^{1 / 4}, 0.75\right)$ (solid lines) and the leading order small- $k$ asymptotic solution given by (4.49) (dashed line). (b) Plots of $z_{\text {pen }}$ as a function of $\ln k$ given by (3.14)-(3.17) subject to $\left(r_{0}, z_{0}\right)=(0.5,0.25),(0.5,0.5)$ and $(0.5,0.75)$ (solid lines) and the leading order large- $k$ asymptotic solution given by (B.18) (dashed lines). (c) Plots of $\ln \left|z_{\text {pen }}-z_{0}\right|$ as a function of $\ln k$ given by (3.16) and (3.17) subject to $\left(r_{0}, z_{0}\right)=(0.5,-0.25),(0.5,-0.5)$ and $(0.5,-0.75)$ (solid lines) and the leading order small $k$ asymptotic solution given by (4.54) (dashed lines). (d) Plots of $z_{\text {pen }}$ as a function of $\ln k$ given by (3.16) and (3.17) subject to $\left(r_{0}, z_{0}\right)=(0.5,-0.25),(0.5,-0.5)$ and $(0.5,-0.75)$ (solid lines) and the leading order large- $k$ asymptotic solution given by (B.21) (dashed lines). In all of the plots $\alpha=1$ and $\phi=3 / 4$. 
$\left|z_{\text {pen }}-z_{0}\right|=O\left(k^{1 / 3}\right) \ll 1$ of a fluid particle initially situated in the porous bed is small and decreases monotonically as $z_{0}$ decreases.) Figure 8(d) shows plots of $z_{\text {pen }}$ as a function of $\ln k$ for "large" values of the permeability $k$ for three fluid particles initially situated in the porous bed. In particular, Figure 8(d) shows that, like in Figure 8(b), $\left|z_{\text {pen }}\right|$ decreases as $k$ decreases and/or $z_{0}$ increases. Moreover, the effect of $k$ on $z_{\text {pen }}$ becomes less pronounced as $k$ increases. (In fact, it will be shown in Appendix B that, as for a fluid particle initially situated in the fluid layer, at leading order in the limit of large permeability $k \rightarrow \infty$ the penetration depth $z_{\mathrm{pen}}=O(1)$ of a fluid particle initially situated in the porous bed is independent of $k$ and its magnitude decreases monotonically as $z_{0}$ increases.)

As we have already seen, there is qualitatively different behaviour for small and large values of the permeability $k$, and so in $\S 4$ we investigate the asymptotic behaviour of the solution in the limit $k \rightarrow 0$, while in Appendix B we investigate the asymptotic behaviour of the solution in the less physically relevant limit $k \rightarrow \infty$.

\section{Limit of Small Permeability $k \rightarrow 0$}

In this section asymptotic solutions for the fluid layer thickness $h=h(t)(\S 4.1)$ and the fluid particle paths $(r, z)(\S 4.2)$ in the limit of small permeability $k \rightarrow 0$ are obtained.

In the limit of small permeability it will transpire that the contact time $t_{\mathrm{c}}=O\left(k^{-2 / 3}\right) \gg 1$ is large, and a regular perturbation solution for $h=h(t)$ in powers of $k^{1 / 2} \ll 1$ turns out to be valid only for "short" times $t=O(1)$ (more precisely, for $t=o\left(k^{-1 / 2}\right)$ ), and additional asymptotic solutions valid for "intermediate" times $t=O\left(k^{-1 / 2}\right)$ and "long" times $t=O\left(k^{-2 / 3}\right)$ are required in order to obtain a complete description of the behaviour up to the contact time.

\subsection{Asymptotic Solution for $h=h(t)$}

4.1.1 Solution for "Short" Times $t=O(1)$ Seeking a regular perturbation solution to (3.2) with $L=L_{0}$ of the form

$$
h(t)=h_{0}(t)+k^{1 / 2} h_{1}(t)+k h_{2}(t)+O\left(k^{3 / 2}\right),
$$

at $O(1), O\left(k^{1 / 2}\right)$ and $O(k)$ we have

$$
\begin{gathered}
\frac{\mathrm{d} h_{0}}{\mathrm{~d} t}=-\frac{2 L_{0} h_{0}^{3}}{3 \pi}, \\
\frac{\mathrm{d} h_{1}}{\mathrm{~d} h_{0}}-\frac{3}{h_{0}} h_{1}=\frac{3}{\alpha h_{0}}, \\
\frac{\mathrm{d} h_{2}}{\mathrm{~d} h_{0}}-\frac{3}{h_{0}} h_{2}=\frac{3\left[h_{0}\left(\alpha^{2} h_{1}^{2}+2 \alpha h_{1}+2 \delta \alpha^{2}-1\right)+4 \alpha^{2}\right]}{\alpha^{2} h_{0}^{3}},
\end{gathered}
$$

respectively, where $t$ has been eliminated from (4.3) and (4.4) using (4.2). Solving (4.2)-(4.4) subject to the initial conditions $h_{0}(0)=d, h_{1}(0)=0$ and $h_{2}(0)=0$ yields the solutions

$$
\begin{gathered}
h_{0}=\left(\frac{3 \pi d^{2}}{3 \pi+4 d^{2} L_{0} t}\right)^{1 / 2}, \\
h_{1}=-\frac{\left(d^{3}-h_{0}^{3}\right)}{\alpha d^{3}}, \\
h_{2}=\frac{3\left[5 h_{0}^{7}+\left(5 \delta \alpha^{2} d^{2}+8 \alpha^{2} d-10 d^{2}\right) h_{0}^{5}+5\left(1-\delta \alpha^{2}\right) d^{6} h_{0}-8 \alpha^{2} d^{6}\right]}{10 \alpha^{2} d^{6} h_{0}^{2}} .
\end{gathered}
$$


The leading order solution for $h_{0}$ given by (4.5) is simply the solution for an impermeable bed given by (3.9), i.e., as expected, the porous bed is effectively impermeable at leading order. The effect of permeability first appears at first order and, since $h_{1}$ given by (4.6) is negative for all $t>0$, its effect is, as expected, to decrease the time $t$ taken for the fluid layer to reduce to a thickness $h$. However, since

$$
h_{0} \sim\left(\frac{3 \pi}{4 L_{0} t}\right)^{1 / 2} \rightarrow 0^{+}, \quad h_{1} \rightarrow-\frac{1}{\alpha}, \quad h_{2} \sim-\frac{16 L_{0} t}{5 \pi} \rightarrow-\infty \quad \text { as } \quad t \rightarrow \infty,
$$

the expansion (4.1) is not uniformly valid for all $t$. Comparing the leading and first order terms suggests that the expansion remains uniformly valid provided that

$$
t \ll \frac{\alpha^{2}}{L_{0} k}, \quad \text { i.e. } \quad t=o\left(k^{-1}\right) .
$$

However, comparing the first and second order terms shows that in fact the expansion remains uniformly valid only provided that

$$
t \ll \frac{1}{L_{0} \alpha k^{1 / 2}}, \quad \text { i.e. } \quad t=o\left(k^{-1 / 2}\right),
$$

and hence a second asymptotic solution valid for "intermediate" times $t=O\left(k^{-1 / 2}\right)$ for which $h=$ $O\left(k^{1 / 4}\right)$ is necessary.

4.1.2 Solution for "Intermediate" Times $t=O\left(k^{-1 / 2}\right) \quad$ For "intermediate" times $t=O\left(k^{-1 / 2}\right)$ we re-scale $t$ and $h$ according to

$$
t=k^{-1 / 2} \hat{t}, \quad h=k^{1 / 4} \hat{h},
$$

where $\hat{t}$ and $\hat{h}$ are $O(1)$ in the limit $k \rightarrow 0$. Seeking a regular perturbation solution to (3.2) with $L=L_{0}$ of the form

$$
\hat{h}(\hat{t})=\hat{h}_{0}(\hat{t})+k^{1 / 4} \hat{h}_{1}(\hat{t})+O\left(k^{1 / 2}\right),
$$

at $O(1)$ and $O\left(k^{1 / 4}\right)$ we have

$$
\begin{gathered}
\frac{\mathrm{d} \hat{h}_{0}}{\mathrm{~d} \hat{t}}=-\frac{2 L_{0} \hat{h}_{0}^{3}}{3 \pi} \\
\frac{\mathrm{d} \hat{h}_{1}}{\mathrm{~d} \hat{h}_{0}}-\frac{3}{\hat{h}_{0}} \hat{h}_{1}=\frac{3\left(4 \alpha+\hat{h}_{0}^{2}\right)}{\alpha \hat{h}_{0}^{3}},
\end{gathered}
$$

respectively. Solving (4.13) and (4.14) subject to the appropriate matching conditions with the solution at "short" times, namely

$$
\hat{h}_{0} \sim\left(\frac{3 \pi}{4 L_{0} \hat{t}}\right)^{1 / 2} \rightarrow \infty, \quad \hat{h}_{1} \rightarrow-\frac{1}{\alpha} \quad \text { as } \quad \hat{t} \rightarrow 0
$$

yields the solutions

$$
\begin{aligned}
& \hat{h}_{0}=\left(\frac{3 \pi}{4 L_{0} \hat{t}}\right)^{1 / 2}, \\
& \hat{h}_{1}=-\frac{12 \alpha+5 \hat{h}_{0}^{2}}{5 \alpha \hat{h}_{0}^{2}} .
\end{aligned}
$$


This solution for "intermediate" times is qualitatively similar to that for "short" times. However, since

$$
\hat{h}_{0}=\left(\frac{3 \pi}{4 L_{0} \hat{t}}\right)^{1 / 2} \rightarrow 0^{+}, \quad \hat{h}_{1} \sim-\frac{16 L_{0} \hat{t}}{5 \pi} \rightarrow-\infty \quad \text { as } \quad \hat{t} \rightarrow \infty
$$

now the expansion remains uniformly valid provided that

$$
\hat{t} \ll \frac{1}{L_{0} k^{1 / 6}}, \quad \text { i.e. } \quad t \ll \frac{1}{L_{0} k^{2 / 3}},
$$

and hence a third (and final) asymptotic solution valid for "long" times $t=O\left(k^{-2 / 3}\right)$ for which $h=$ $O\left(k^{1 / 3}\right)$ is necessary.

4.1.3 Solution for "Long" Times $t=O\left(k^{-2 / 3}\right) \quad$ For "long" times $t=O\left(k^{-2 / 3}\right)$ we re-scale $t$ and $h$ according to

$$
t=(12 k)^{-2 / 3} T, \quad h=(12 k)^{1 / 3} H
$$

where $T$ and $H$ are $O(1)$ in the limit $k \rightarrow 0$ and the factor 12 has been introduced into the scalings for convenience. Seeking a regular perturbation solution to (3.2) with $L=L_{0}$ of the form

$$
H(T)=H_{0}(T)+(12 k)^{1 / 6} H_{1}(T)+O\left(k^{1 / 3}\right),
$$

at $O(1)$ and $O\left(k^{1 / 6}\right)$ we have

$$
\begin{gathered}
\frac{\mathrm{d} H_{0}}{\mathrm{~d} T}=-\frac{2 L_{0}\left(H_{0}^{3}+1\right)}{3 \pi}, \\
\frac{\mathrm{d} H_{1}}{\mathrm{~d} H_{0}}-\frac{3 H_{0}^{2}}{H_{0}^{3}+1} H_{1}=\frac{\sqrt{3} H_{0}^{2}}{2 \alpha\left(H_{0}^{3}+1\right)},
\end{gathered}
$$

respectively. Solving (4.22) and (4.23) subject to the appropriate matching conditions with the solution at "intermediate" times, namely

$$
H_{0} \sim\left(\frac{3 \pi}{4 L_{0} T}\right)^{1 / 2} \rightarrow \infty, \quad H_{1} \rightarrow-\frac{1}{2 \sqrt{3} \alpha} \quad \text { as } \quad T \rightarrow 0,
$$

yields the solutions

$$
\begin{gathered}
T=\frac{\pi\left[f(\infty)-f\left(H_{0}\right)\right]}{2 L_{0}}, \\
H_{1}=-\frac{1}{2 \sqrt{3} \alpha},
\end{gathered}
$$

where the monotonically increasing function $f=f\left(H_{0}\right)$ is given by

$$
f\left(H_{0}\right)=\ln \left(\frac{H_{0}+1}{\sqrt{H_{0}^{2}-H_{0}+1}}\right)+\sqrt{3} \tan ^{-1}\left(\frac{2 H_{0}-1}{\sqrt{3}}\right)
$$

and satisfies $f(0)=-\sqrt{3} \pi / 6$ and $f(\infty)=\sqrt{3} \pi / 2$. Unlike for the solutions for "short" and "intermediate" times, the permeability now appears in the leading order solution and the solution remains 
uniformly valid up to the contact time $t=t_{\mathrm{c}}$ given by $t_{\mathrm{c}}=(12 \mathrm{k})^{-2 / 3} T_{\mathrm{c}}$, where $T_{\mathrm{c}}$ satisfies $H\left(T_{\mathrm{c}}\right)=0$, and has the asymptotic expansion

$$
T_{\mathrm{c}}=T_{\mathrm{c} 0}+(12 k)^{1 / 6} T_{\mathrm{c} 1}+O\left(k^{1 / 3}\right)
$$

where

$$
H_{0}\left(T_{\mathrm{c} 0}\right)=0, \quad T_{\mathrm{c} 1}=-\frac{H_{1}\left(T_{\mathrm{c} 0}\right)}{H_{0}^{\prime}\left(T_{\mathrm{c} 0}\right)}
$$

so that

$$
T_{\mathrm{c} 0}=\frac{\pi^{2}}{\sqrt{3} L_{0}}, \quad T_{\mathrm{c} 1}=-\frac{\sqrt{3} \pi}{4 L_{0} \alpha}
$$

Expressed in terms of the original variables the asymptotic expansion for the contact time (4.28) is therefore

$$
t_{\mathrm{c}}=\frac{\pi^{2}}{\sqrt{3} L_{0}(12 k)^{2 / 3}}-\frac{\pi}{8 L_{0} \alpha k^{1 / 2}}+O\left(k^{-1 / 3}\right)
$$

In particular, the leading order small- $k$ solution for the contact time given by

$$
t_{\mathrm{c}}=\frac{\pi^{2}}{\sqrt{3} L_{0}(12 k)^{2 / 3}}
$$

is independent of both $\alpha$ and $\delta$, and so is the same for all three choices of boundary condition (2.8)(2.10). Figure 2(b) compares the leading order small- $k$ asymptotic solution for $t_{\mathrm{c}}$ given by (4.32) with the exact solution given by (3.11). The Beavers-Joseph constant $\alpha$ appears at first order in the asymptotic expansion for $t_{\mathrm{c}}$ given by (4.31), and inspection of the first order term reveals that decreasing $\alpha$ (i.e. increasing the slip length $l_{\mathrm{s}}$ ) decreases the contact time $t_{\mathrm{c}}$, consistent with the behaviour shown in Figure 3(a).

4.1.4 A Uniformly Valid Leading Order Composite Solution for t $(h) \quad$ A uniformly valid leading order composite solution for $t=t(h)$ is

$$
t(h)=-\frac{3 \pi}{4 d^{2} L_{0}}+(12 k)^{-2 / 3} T\left(\frac{h}{(12 k)^{1 / 3}}\right),
$$

where $T$ is given by (4.25). Figures 2(a) and 3(a) compare the uniformly valid leading order small- $k$ asymptotic solution for $t=t(h)$ given by (4.33) with the exact solution for $t=t(h)$ given by (3.8). (Note that setting $h=d$ in (4.33) yields $t(d)=O(k)$ as $k \rightarrow 0$, so that (4.33) satisfies the initial condition (2.31) at leading order in the limit $k \rightarrow 0$. Similarly, setting $h=0$ in (4.33) yields $t(0) \sim \pi^{2} / \sqrt{3} L_{0}(12 k)^{2 / 3}$ as $k \rightarrow 0$, so that (4.33) coincides with (4.32) at leading order in the limit $k \rightarrow 0$.)

\subsection{Asymptotic Solution for the Fluid Particle Paths}

4.2.1 Fluid Particles Initially Situated in the Fluid Layer $z_{0}>0$ The analysis in $\S 4.1$ shows that in the limit of small permeability $k \rightarrow 0$ the porous bed is effectively impermeable for "short" and "intermediate" times, i.e. for $t=o\left(k^{-2 / 3}\right)$, and so at leading order the paths of fluid particles initially 
situated in the fluid layer (i.e. with $z_{0}>0$ ) are given by the solution for an impermeable bed given by (3.20) and (3.21). When $t=O\left(k^{-2 / 3}\right)$ and $h=O\left(k^{1 / 3}\right)$ the solution for an impermeable bed yields

$$
\begin{aligned}
& r \sim r_{0}\left(\frac{4\left(d-z_{0}\right) z_{0}}{d h}\right)^{3 / 4}=O\left(\frac{r_{0}\left(\left(d-z_{0}\right) z_{0}\right)^{3 / 4}}{k^{1 / 4}}\right), \\
& z \sim \frac{h}{2}=O\left(k^{1 / 3}\right),
\end{aligned}
$$

showing that only those fluid particles whose initial positions are close to the axis $r=0$ (specifically, those with $r_{0}=O\left(k^{1 / 4}\right)$ ) or are close to either the porous bed or the bearing (specifically, those with $z_{0}=O\left(k^{1 / 3}\right)$ or $d-z_{0}=O\left(k^{1 / 3}\right)$, respectively) have not flowed out of the fluid layer into the region beyond $r=1$ at "long" times.

In order to analyse the path of a fluid particle initially situated in the fluid layer close to the axis $r=0$ with $r_{0}=O\left(k^{1 / 4}\right)$ and $z_{0}>0$ when $t=O\left(k^{-2 / 3}\right)$ and $h=O\left(k^{1 / 3}\right)$, we re-scale $r_{0}, z, t$ and $h$ according to

$$
r_{0}=(12 k)^{1 / 4} R_{0}, \quad z=(12 k)^{1 / 3} Z, \quad t=(12 k)^{-2 / 3} T, \quad h=(12 k)^{1 / 3} H,
$$

where $R_{0}, Z, T$ and $H$ are $O(1)$ in the limit $k \rightarrow 0$ and the factor 12 has again been introduced into the scalings for convenience.

From (3.14) and (3.15) the leading order equations for the path of a fluid particle in the fluid layer are

$$
\begin{aligned}
\frac{1}{r} \frac{\mathrm{d} r}{\mathrm{~d} H} & =-\frac{3(H-Z) Z}{H^{3}+1}, \\
\frac{\mathrm{d} Z}{\mathrm{~d} H} & =\frac{-2 Z^{3}+3 H Z^{2}+1}{H^{3}+1} .
\end{aligned}
$$

The solutions to (4.37) and (4.38) must match with the $h \rightarrow 0$ limits of (3.20) and (3.21) as $H \rightarrow \infty$, namely

$$
\begin{aligned}
r & \sim R_{0}\left(\frac{4\left(d-z_{0}\right) z_{0}}{d H}\right)^{3 / 4} \rightarrow 0 \quad \text { as } \quad H \rightarrow \infty \\
Z & \sim \frac{H}{2} \rightarrow \infty \quad \text { as } \quad H \rightarrow \infty
\end{aligned}
$$

The leading order vertical position of the fluid particle $Z(T)$, which is independent of the initial height of the fluid particle $z_{0}$, was obtained numerically by posing (4.38) as an initial value problem by shooting from the value $Z=0$ at the unknown value of the fluid layer thickness $H=H^{*}$ at which the fluid particle crosses the interface $Z=0$ to $Z=H_{\infty} / 2$ at some large value $H=H_{\infty} \gg 1$ approximating the matching condition (4.40) to within a prescribed tolerance, namely $10^{-6}$. This procedure yielded the solution $H^{*} \simeq 0.4958$ corresponding to $T=T^{*}=T\left(H^{*}\right)$, which from (4.20) and (4.25) corresponds to the time

$$
t=t^{*}=(12 k)^{-2 / 3} T\left(H^{*}\right) \simeq \frac{0.6540}{L_{0} k^{2 / 3}}
$$

The leading order radial position of the fluid particle $R(T)$ is calculated by integrating (4.37) from $H=H^{*}$ to $H$ to obtain $r=r^{*} g(H)$, where $r=r^{*}$ is again the radial position at which the fluid particle 


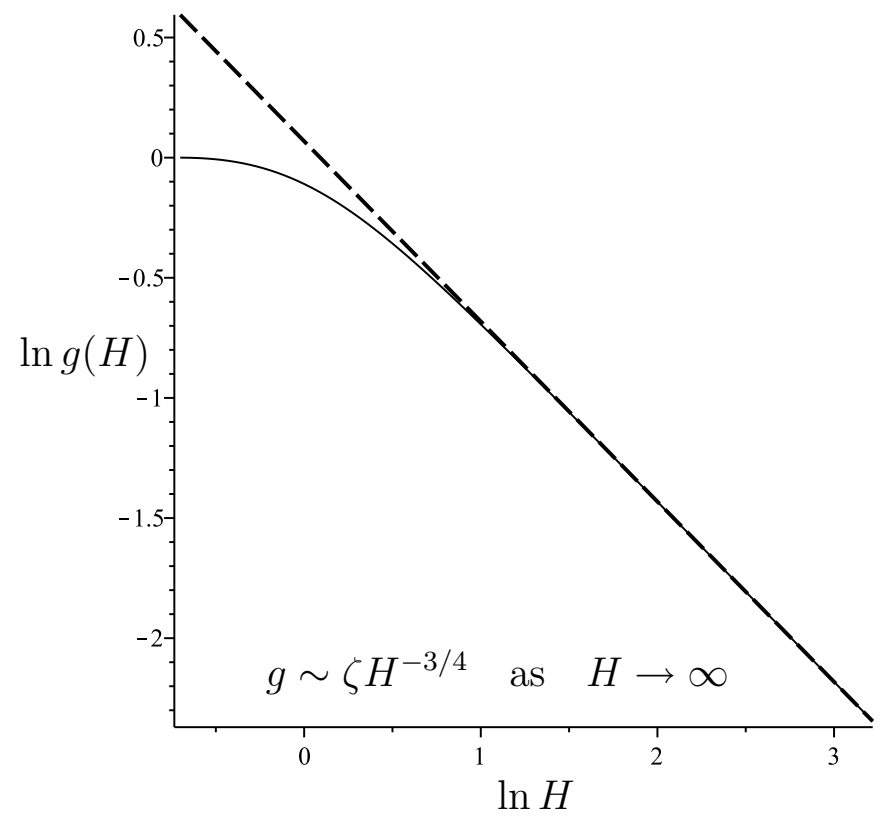

FIG. 9. Plots of $\ln g(H)$ as a function of $\ln H$ given by (4.42) (solid line) and its large- $H$ asymptotic behaviour $g \sim \zeta H^{-3 / 4}$, where $\zeta \simeq 1.0708$ (dashed line).

crosses the interface $Z=0$ and the monotonically decreasing function $g=g(H)$ is defined by

$$
g(H)=\exp \left[-3 \int_{H^{*}}^{H} \frac{(s-Z) Z}{s^{3}+1} \mathrm{~d} s\right] .
$$

Figure 9 shows a plot of $\ln g(H)$ as a function of $\ln H$ together with its large- $H$ asymptotic behaviour $g \sim \zeta H^{-3 / 4}$, where $\zeta \simeq 1.0708$. Imposing the matching condition (4.39) yields

$$
r^{*}=\frac{R_{0}}{\zeta}\left(\frac{4\left(d-z_{0}\right) z_{0}}{d}\right)^{3 / 4}
$$

In particular, the leading order solution for the critical curve $r^{*}=1$ is therefore given by

$$
r_{0}=\zeta\left(\frac{d}{4\left(d-z_{0}\right) z_{0}}\right)^{3 / 4}(12 k)^{1 / 4}
$$

Figure 7 compares the leading order small- $k$ asymptotic solution (4.44) with the exact solution for $r^{*}=1$ for $k=10^{-4}$. In particular, it shows that the asymptotic solution compares favourably with the exact solution when $z_{0}=O(1)$. However, as Figure 7 also shows, the asymptotic solution fails for $z_{0}=O\left(k^{1 / 3}\right) \ll 1$ and for $d-z_{0}=O\left(k^{1 / 3}\right) \ll 1$, i.e. for fluid particles that are initially close to either the porous bed or the bearing; for brevity these cases are not considered further here.

From (3.16) and (3.17) the leading order equations for the path of a fluid particle after it has entered 
the porous bed at $r=r^{*}$ are

$$
\begin{aligned}
\frac{\mathrm{d} R}{\mathrm{~d} H} & =-\frac{r^{*}}{2 \phi\left(H^{3}+1\right)}, \\
\frac{\mathrm{d} Z}{\mathrm{~d} H} & =\frac{1}{\phi\left(H^{3}+1\right)},
\end{aligned}
$$

where we have written $r=r^{*}+(12 k)^{1 / 3} R$. Solving (4.45) and (4.46) subject to the initial conditions $R=0$ and $Z=0$ at $H=H^{*}$ yields

$$
\begin{aligned}
& r=r^{*}\left(1+\frac{\left[f\left(H^{*}\right)-f(H)\right](12 k)^{1 / 3}}{6 \phi}\right), \\
& z=-\frac{\left[f\left(H^{*}\right)-f(H)\right](12 k)^{1 / 3}}{3 \phi}
\end{aligned}
$$

for $0 \leqslant H<H^{*}$, where the function $f=f(H)$ is given by (4.27) and satisfies $f\left(H^{*}\right) \simeq 0.5381$, showing that the fluid particle remains close to $r=r^{*}$ and $z=0$. In particular, setting $H=0$ in (4.48) shows that the leading order asymptotic solution for $z_{\text {pen }}$ is

$$
z_{\mathrm{pen}}=-\frac{\left[f\left(H^{*}\right)-f(0)\right](12 k)^{1 / 3}}{3 \phi} \simeq-\frac{1.1027 k^{1 / 3}}{\phi}
$$

which depends on $k$ but is independent of $z_{0}$. Figure 8(a) compares the leading order small- $k$ asymptotic solution for $z_{\text {pen }}$ given by (4.49) with the exact solution.

Figure 10 compares the scaled fluid particle paths in (a) the fluid layer and (b) the porous bed with the leading order small- $k$ asymptotic solution given by $r=r^{*} g(H)$ and the numerical solution of (4.38) for $H \geqslant H^{*}$, and by (4.47) and (4.48) for $0 \leqslant H<H^{*}$.

4.2.2 Fluid Particles Initially Situated in the Porous Bed $z_{0}<0 \quad$ As we have already seen, the analysis in $\S 4.1$ shows that in the limit of small permeability $k \rightarrow 0$ the porous bed is effectively impermeable for "short" and "intermediate" times, i.e. for $t=o\left(k^{-2 / 3}\right)$, and so at leading order fluid particles initially situated in the porous bed (i.e. with $z_{0}<0$ ) remain close to their initial positions, specifically

$$
\begin{aligned}
& r=r_{0}\left(1+\frac{3\left(d^{2}-h^{2}\right) k}{\phi d^{2} h^{2}}\right), \\
& z=z_{0}-\left(1+z_{0}\right) \frac{6\left(d^{2}-h^{2}\right) k}{\phi d^{2} h^{2}} .
\end{aligned}
$$

For "long" times, i.e. for $t=O\left(k^{-2 / 3}\right)$, a similar analysis to that described previously in $\S 4.2 .1$ yields

$$
\begin{aligned}
& r=r_{0}\left(1+\frac{[f(\infty)-f(H)](12 k)^{1 / 3}}{6 \phi}\right) \\
& z=z_{0}-\left(1+z_{0}\right) \frac{[f(\infty)-f(H)](12 k)^{1 / 3}}{3 \phi},
\end{aligned}
$$


(a)

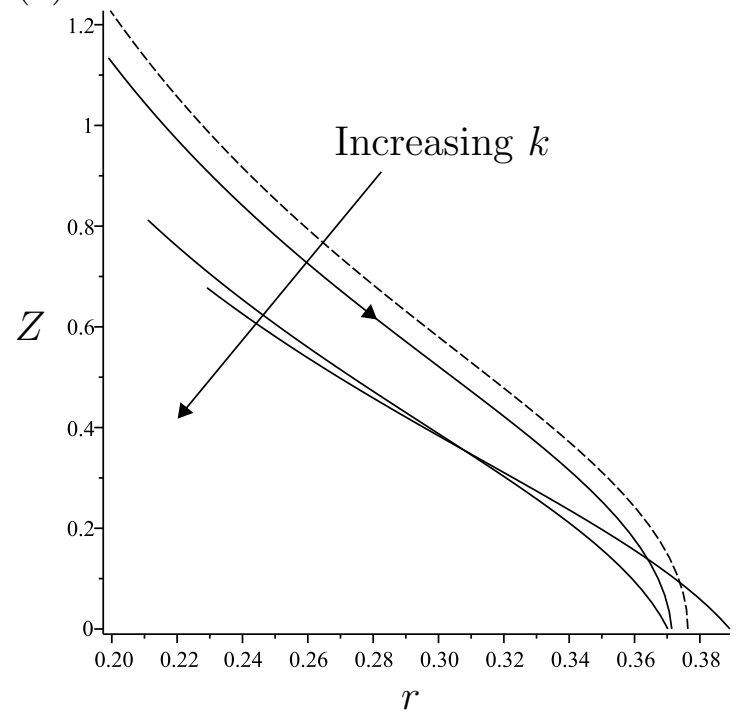

(b)

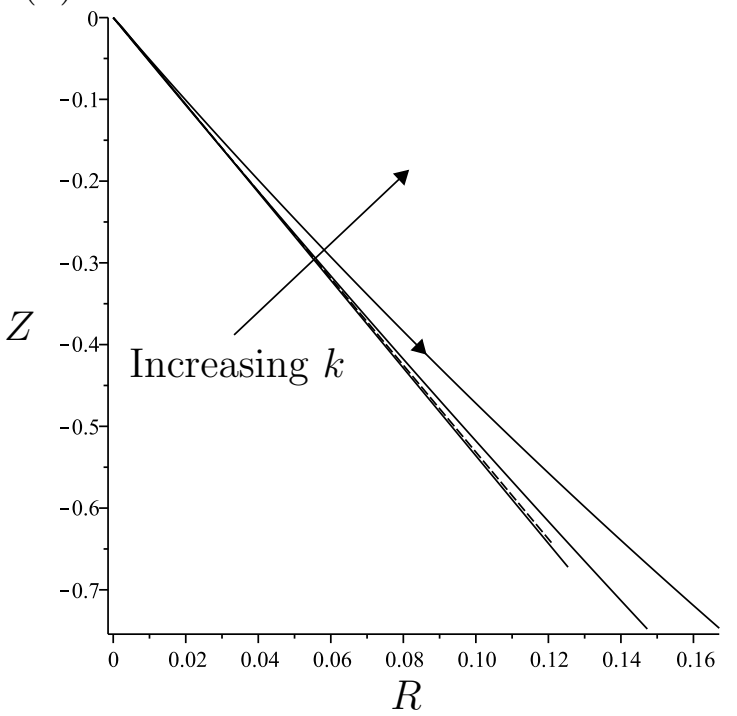

FIG. 10. (a) Plots of the scaled fluid particle paths $(r, Z)$ in the fluid layer, where $Z=z /(12 k)^{1 / 3}$, given by (3.14)-(3.17) subject to $\left(r_{0}, z_{0}\right)=\left(0.5 \times(12 k)^{1 / 4}, 0.25\right)$ for $k=10^{-3}, 10^{-4}$ and $10^{-8}$ (solid lines) and the leading order small- $k$ asymptotic solution given by $r=r^{*} g(H)$ and the numerical solution of (4.38) for $H \geqslant H^{*}$ (dashed line). (b) Plots of the scaled fluid particle paths $(R, Z)$ in the porous bed, where $R=\left(r-r^{*}\right) /(12 k)^{1 / 3}$ and $Z=z /(12 k)^{1 / 3}$, given by $(3.14)-(3.17)$ subject to $\left(r_{0}, z_{0}\right)=\left(0.5 \times(12 k)^{1 / 4}, 0.25\right)$ for $k=10^{-3}, 10^{-4}$ and $10^{-8}$ and the leading order small- $k$ asymptotic solution given by (4.47) and (4.48) for $0 \leqslant H<H^{*}$ (dashed line). In all of the plots $\alpha=1$ and $\phi=3 / 4$. 
(a)

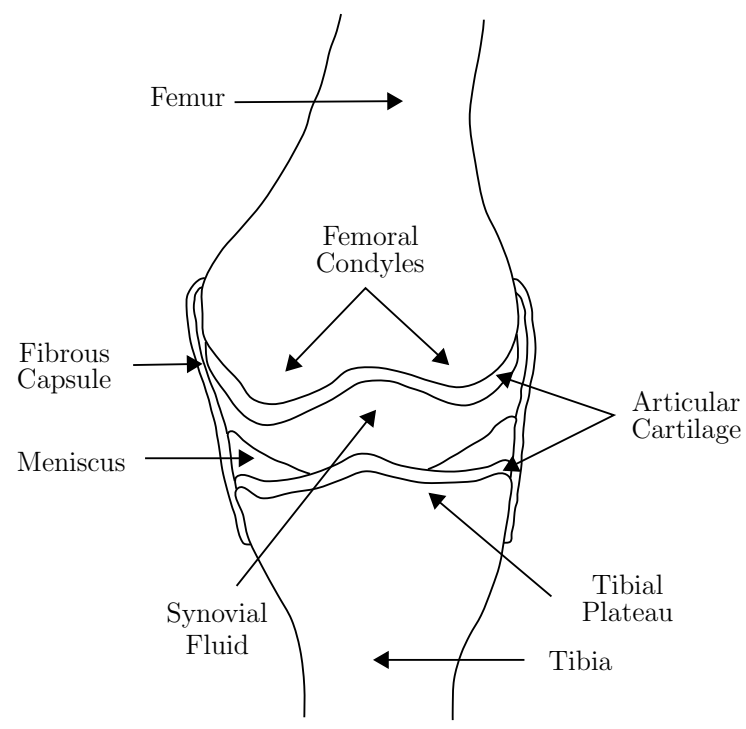

(b)

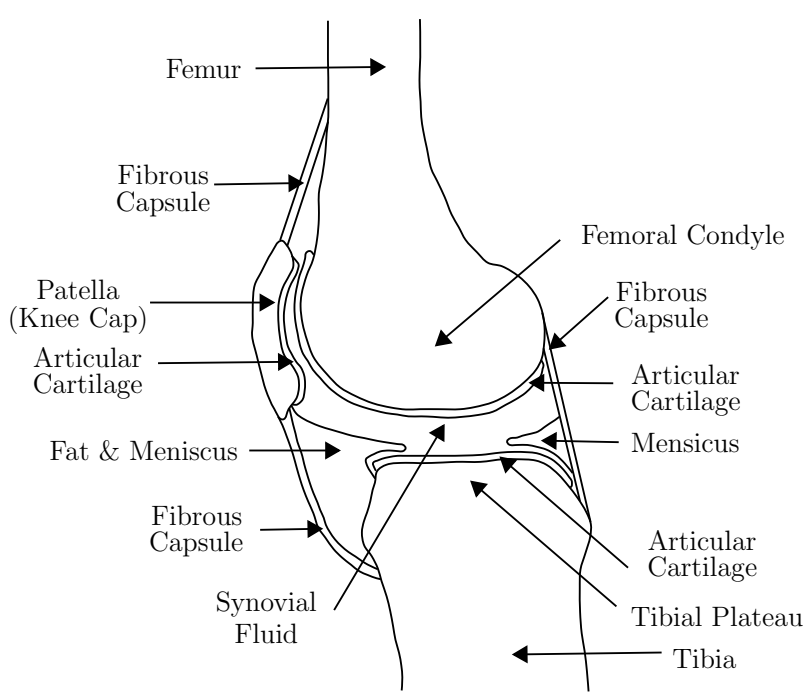

FIG. 11. Sketches of the main anatomical features of the human knee joint. A slice through (a) the front of the knee (behind the patella) and (b) the side of the knee (through the patella).

$$
z_{\mathrm{pen}}=z_{0}-\left(1+z_{0}\right) \frac{[f(\infty)-f(0)](12 k)^{1 / 3}}{3 \phi}=z_{0}-\frac{2 \pi\left(1+z_{0}\right)(12 k)^{1 / 3}}{3 \sqrt{3} \phi},
$$

which depends on $k$ and $z_{0}$. Figure 8(c) compares the leading order small- $k$ asymptotic solution for $z_{\text {pen }}$ given by (4.54) with the exact solution.

\section{Conclusions and Application to the Human Knee Joint}

In the present work we considered the squeeze-film flow of a thin layer of Newtonian fluid filling the gap between a flat impermeable surface moving under a prescribed constant load and a flat thin porous bed coating a stationary flat impermeable surface. Using a lubrication approximation an implicit expression for the fluid layer thickness $h=h(t)$ and an explicit expression for the contact time $t_{\mathrm{c}}$ were obtained and analysed. In addition, the fluid particle paths were calculated, and the penetration depths of fluid particles into the porous bed were determined. In particular, the behaviour in the asymptotic limit of small permeability, $k \rightarrow 0$, in which the contact time $t_{\mathrm{c}}=O\left(k^{-2 / 3}\right) \gg 1$ is large but finite, was investigated.

Our interest in porous squeeze-film flow was originally motivated by the desire for a better understanding of the lubrication of the human knee joint, and so in the remainder of the present paper we seek to interpret the results obtained in this context. As well as being of fundamental scientific and medical interest in its own right, understanding the lubrication of the knee is of crucial importance in the development of total-knee-replacement technologies (see, for example, Walker et al. (2010)).

Figure 11 shows sketches of the main anatomical features of the human knee joint. The knee is where the largest bones in the human body, the femur and the tibia, meet. Situated at the distal (furthest 
from the torso) end of the femur are two rounded "knobs" which are referred to as the femoral condyles. When the knee is in flexion or extension the femoral condyles articulate with the tibial plateau which is situated at the proximal (closest to the torso) end of the tibia. Both the femoral condyles and the tibial plateau are coated with a thin layer of articular cartilage, which is a poroelastic material, and the gap between the femoral condyles and the tibial plateau is lubricated by a thin layer of synovial fluid. The latter is a non-Newtonian shear-thinning fluid and has an egg-white-like consistency (see, for example, Fam et al. (2007) and Balazs (2009)). Two crescent-shaped wedges of cartilage, the lateral and medial menisci, are situated on the peripheral boundary of the tibial plateau. The menisci deepen the surface of the tibial plateau, protecting the underlying articular cartilage and stabilising the joint, and are attached to the fibrous capsule, which is a collection of ligaments that surround the joint, connecting the tibia with the femur, and the patella (knee cap) with both the tibia and the femur. The fibrous capsule prevents the joint from undergoing any abnormal movements, and is lined by the synovial membrane, which secretes and absorbs the synovial fluid that lubricates the joint. The latest (40th) edition of Gray's Anatomy (Standring (2008)) contains an excellent overview of the knee.

When a person walks or stands still the force $L=L(t)$ acting through the knee pushes the femoral condyles and the tibial plateau together when $L>0$, creating a squeeze-film effect, and pulls them apart when $L<0$. The synovial fluid may be squeezed into or sucked out of the cartilage. Cartilage is avascular (i.e. it does not have a blood supply) and so the flow of synovial fluid into the cartilage is one of the ways that it receives nutrients (see, for example, O'Hara et al. (1990)). It is believed that when a person walks a thin layer of synovial fluid, reported to be up to $5 \times 10^{-7} \mathrm{~m}$ thick (see, for example, Stewart et al. (1997)), can separate the tibial plateau and the femoral condyles. However, when a person stands still for an extended period of time it is believed that the synovial fluid will eventually be squeezed out of the gap between the tibial plateau and the femoral condyles, and so the cartilage-coated surfaces will eventually come into contact (see, for example, Stewart et al. (1997)); this is undesirable as it could result in cartilage degradation and promote the onset of osteoarthritis. The recent review article by Ateshian (2009) and the references therein provide a very useful summary of recent thinking on cartilage lubrication.

Various squeeze-film flows of varying degrees of complexity similar to that studied in the present work have been proposed as models for the lubrication of the human knee joint (see, for example, Hou et al. (1992) and Skotheim \& Mahadevan (2005)), and so it is natural to ask what implications the present results have in this context. Obviously modelling the cartilage as a homogenous rigid porous bed (as we do here) is a highly simplistic idealisation of a very complicated biological material. In reality, articular cartilage is a fibrillar matrix of collagen, large aggregating proteoglycans, and chondrocytes (see, for example, Mow \& Ratcliffe (1997)) which exhibits strong compositional heterogeneity and sustains an osmotic pressure higher than that of the external fluid (see, for example, Maroudas (1976)). As a result of the complexity and importance of cartilage, a number of mathematical models for various aspects of it of varying degrees of sophistication, notably the linear biphasic theory of Mow et al. (1980) (see the review article by Mow \& Guo (2002) and the references therein), have been proposed and analysed. Nevertheless there is some value in using the present highly idealised model to investigate some of the fluid mechanical aspects of the problem, namely the contact time of the cartilage-coated femoral condyles and tibial plateau and the penetration of nutrients into the cartilage. However, before doing this, two further simplifications inherent in the present model require some further justification.

First, modelling the femoral condyles as a flat bearing appears to be overly simplistic. However, Weekley et al. (2006), who considered the approach of a parabolic surface towards a flat surface coated with a thin elastic layer through a thin layer of Newtonian fluid, found that the elastic layer deforms in such a way as to create a fluid layer of approximately uniform thickness, with the consequence that 


\begin{tabular}{|c|c|c|c|}
\hline Parameter & Typical Size & Units & Reference \\
\hline$\mu$ & $O\left(10^{-2}\right)$ & Pa s & Stewart et al. $(1997)$ \\
\hline $\mathscr{R}$ & $O\left(10^{-2}\right)$ & $\mathrm{m}$ & Nuño \& Ahmed (2003) \\
\hline$L$ & $O\left(10^{3}\right)$ & $\mathrm{N}$ & Stewart et al. $(1997)$ \\
\hline$H_{\mathrm{p}}$ & $O\left(10^{-3}\right)$ & $\mathrm{m}$ & Ateshian \& Hung (2006) \\
\hline$k$ & $O\left(10^{-18}\right)$ & $\mathrm{m}^{2}$ & Ateshian \& Hung (2006) \\
\hline
\end{tabular}

Table 1. Typical sizes of the dimensional parameters appearing in the model relevant to the human knee joint.

the approach is qualitatively the same as that of two flat rigid surfaces. Since the femoral condyles are roughly parabolic and cartilage is poroelastic, we anticipate qualitatively similar behaviour in the knee, and thus modelling the femoral condyles as a flat bearing is not an unreasonable first model.

Second, assuming that only the tibial plateau is coated with cartilage also appears to be overly simplistic. However, when the condition of no slip relative to the porous bed (2.10) is used the problem of a single porous layer of thickness $H_{\mathrm{p}}$ coating the tibial plateau studied in the present work is, at least as far as the motion of the bearing is concerned, mathematically equivalent to the problem of two porous layers each of thickness $H_{\mathrm{p}} / 2$ coating both the femoral condyles and the tibial plateau, and so the assumption is not as unrealistic as it might at first appear. When either the Beavers-Joseph condition (2.8) or the condition of no slip relative to the fluid (2.9) is used the two problems are not strictly mathematically equivalent but, since we have shown that in the limit of small permeability the leading order behaviour is the same as for (2.10), we expect that, in practice, the two problems will have qualitatively similar behaviour.

As we have shown, as the impermeable bearing approaches the porous bed under a prescribed constant load all of the fluid is squeezed out of the gap in a finite contact time $t_{\mathrm{c}}$. Typical sizes of the dimensional parameters appearing in the model relevant to the human knee joint are given in Table 1. Note that the porosity $\phi$ is not included in Table 1 as it is, by definition, dimensionless and $O(1)$. Ateshian \& Hung (2006) state that for the cartilage in an adult knee $\phi$ lies typically between 0.68 and 0.85 . In particular, Table 1 shows that the non-dimensional permeability $k=O\left(10^{-12}\right)$ is typically very small, meaning that the limit of small permeability $k \rightarrow 0$ is most relevant to the human knee. In this limit we found that the leading order asymptotic solution for $t_{\mathrm{c}}$ is given by (4.32), which is independent of the choice of boundary condition (2.8)-(2.10) and is expressed in terms of dimensional variables as

$$
t_{\mathrm{c}}=\frac{\pi^{2} \mu \mathscr{R}^{4}}{\sqrt{3} L\left(12 H_{\mathrm{p}} k\right)^{2 / 3}}=O(10) \mathrm{s} .
$$

Hence the present model predicts that when a person stands still for a short period of time their knees may be fluid lubricated, but that when they stand still for a longer period of time contact between the cartilage-coated surfaces may occur. Of course, during physical activity such as walking or running a person repeatedly loads and unloads their knees with a temporally varying load. Since typically each step or stride takes around $1 \mathrm{~s}$, which is somewhat less than the typical size of $t_{\mathrm{c}}$ given by (5.1), the present model also suggests that their knees may also remain fluid lubricated during walking or running. Moreover, the corresponding size of $h=O\left(\left(H_{\mathrm{p}} k\right)^{1 / 3}\right)=O\left(10^{-7}\right) \mathrm{m}$ is consistent with the value of $5 \times 10^{-7} \mathrm{~m}$ quoted by Stewart et al. (1997).

We also found that in the limit of small permeability the leading order asymptotic solution for the penetration depth $z_{\text {pen }}$ of fluid particles initially situated in the fluid layer into the porous bed is given by 
(4.49) and is expressed in terms of dimensional variables as

$$
z_{\text {pen }}=-\frac{1.1027\left(H_{\mathrm{p}} k\right)^{1 / 3}}{\phi}=O\left(10^{-7}\right) \mathrm{m}
$$

which is much less than $H_{\mathrm{p}}$. Hence the present model predicts that when a person stands still any nutrients initially in the fluid layer penetrate only a relatively small distance into the cartilage. Moreover, the relative penetration depth $z_{\text {pen }}-z_{0}$ of fluid particles initially situated in the porous bed is given by (4.54) and is expressed in dimensional variables as

$$
z_{\mathrm{pen}}-z_{0}=-\frac{2 \pi\left(H_{\mathrm{p}}+z_{0}\right)\left(12 H_{\mathrm{p}} k\right)^{1 / 3}}{3 \sqrt{3} \phi H_{\mathrm{p}}}=O\left(10^{-7}\right) \mathrm{m},
$$

which is of approximately the same size as $z_{\text {pen }}$ given by (5.2). Hence the present model also predicts that when a person stands still any nutrients initially in the cartilage penetrate only a relatively small distance further into it. However, the cumulative effect of repeated loading and unloading of the knees may alter this conclusion. Assuming that a reasonably active person takes $O\left(10^{4}\right)$ steps per day, the size of the relative penetration depth given in (5.3) corresponds to a maximum cumulative vertical displacement of $O\left(10^{-3}\right) \mathrm{m}$, which is comparable with $H_{\mathrm{p}}$, i.e. the present model also suggests that the cumulative effect of repeated loading and unloading of the knees during physical activity such as walking or running may be sufficient to carry nutrients deep into the cartilage.

\section{Acknowledgements}

The first author (DJK) gratefully acknowledges the financial support of the University of Strathclyde via a University Postgraduate Research Scholarship. Part of this work as undertaken while the corresponding author (SKW) was a Visiting Fellow in the Department of Mechanical and Aerospace Engineering, School of Engineering and Applied Science, Princeton University, USA, and part of it was undertaken while he was a Visiting Fellow in the Oxford Centre for Collaborative Applied Mathematics (OCCAM), Mathematical Institute, University of Oxford, United Kingdom. This publication was based on work supported in part by Award No KUK-C1-013-04, made by King Abdullah University of Science and Technology (KAUST). All four authors gratefully acknowledge valuable discussions with Dr Richard Black, Dr Philip Riches and Mr Anthony Herbert from the Bioengineering Unit (now the Department of Biomedical Engineering) at the University of Strathclyde on the biomechanics of the knee.

\section{A. Appendix A: Two-Dimensional Problem}

The main body of the present work concerns an axisymmetric squeeze-film flow. However, the corresponding two-dimensional problem is also of some interest and may be analysed in the same manner. Accordingly, in this Appendix the main results for the two-dimensional problem shown in Figure 12 are described and the key similarities to and differences from the corresponding results for the axisymmetric problem are highlighted.

Consider, with reference to the two-dimensional Cartesian coordinate system $(x, z)$ shown in Figure 12 , the two-dimensional problem of a bearing of (dimensional) semi-width $\mathscr{L}$ with (dimensional) load $L=L(t)$ per unit width. This problem is non-dimensionalised in the same way as the axisymmetric problem (2.15) with $\mathscr{L}$ in place of $\mathscr{R}$ and $V=L_{0} H_{\mathrm{p}}^{2} /\left(\mu \mathscr{L}^{2}\right)$ in place of $V=L_{0} H_{\mathrm{p}}^{2} /\left(\mu \mathscr{R}^{3}\right)$ as the 


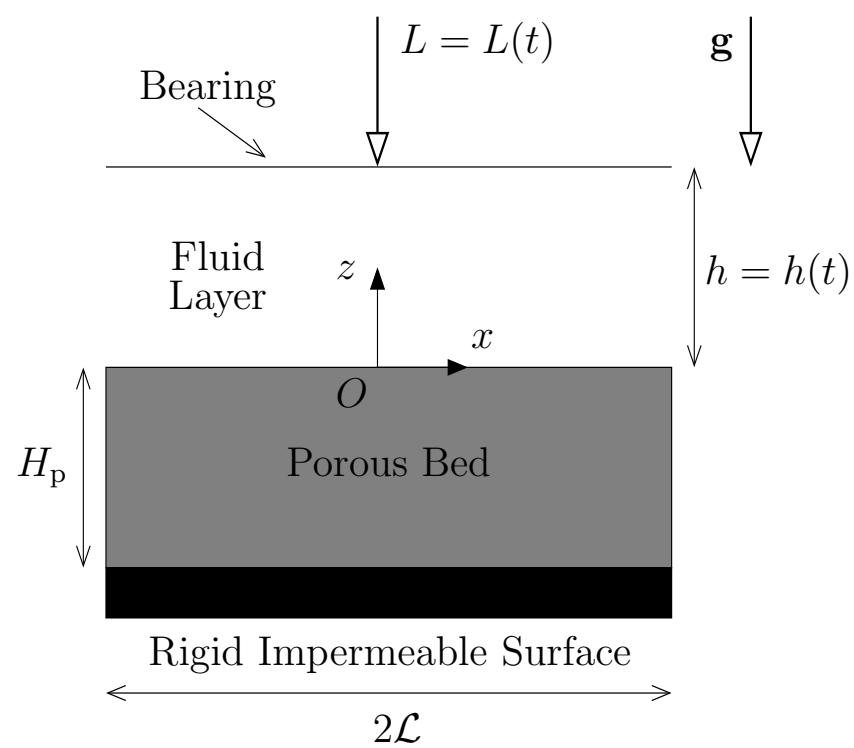

FIG. 12. The geometry of the two-dimensional problem.

characteristic scales for horizontal distance and horizontal fluid velocity, respectively. The horizontal and vertical components of the fluid velocity, $v_{x}=v_{x}(x, z, t)$ and $v_{z}=v_{z}(x, z, t)$, are given by

$$
\begin{aligned}
& v_{x}=-\frac{(h-z)\left[\left(\alpha h+k^{1 / 2}\right) z+k^{1 / 2}\left(h+2 \delta \alpha k^{1 / 2}\right)\right]}{2\left(\alpha h+k^{1 / 2}\right)} \frac{\partial p}{\partial x}, \\
& v_{z}=\frac{2\left(\alpha h+k^{1 / 2}\right)\left(6 k-z^{3}\right)+3 \alpha\left(h^{2}-2 \delta k\right) z^{2}+6 k^{1 / 2} h\left(h+2 \delta \alpha k^{1 / 2}\right) z}{12\left(\alpha h+k^{1 / 2}\right)} \frac{\partial^{2} p}{\partial x^{2}},
\end{aligned}
$$

respectively, the horizontal and vertical components of the Darcy velocity, $u_{x}=u_{x}(x, z, t)$ and $u_{z}=$ $u_{z}(x, z, t)$, are given by

$$
u_{x}=-k \frac{\partial p}{\partial x}, \quad u_{z}=k(1+z) \frac{\partial^{2} p}{\partial x^{2}},
$$

respectively, and the unsteady Reynolds equation governing the pressure $p=p(x, t)$ is

$$
\frac{\partial^{2} p}{\partial x^{2}}=\frac{12\left(\alpha h+k^{1 / 2}\right)}{h^{2}\left(\alpha h^{2}+4 k^{1 / 2} h+6 \delta \alpha k\right)+12 k\left(\alpha h+k^{1 / 2}\right)} \frac{\mathrm{d} h}{\mathrm{~d} t} .
$$

Integrating (A.4) twice with respect to $x$, applying the boundary conditions $p=0$ at $x= \pm 1$, and imposing the load condition

$$
L(t)=2 \int_{0}^{1} p \mathrm{~d} x
$$

yields the pressure

$$
p=\frac{3 L(t)\left(1-x^{2}\right)}{4}
$$


and the differential equation governing the fluid layer thickness $h=h(t)$ :

$$
L(t)=-\frac{8\left(\alpha h+k^{1 / 2}\right)}{h^{2}\left(\alpha h^{2}+4 k^{1 / 2} h+6 \delta \alpha k\right)+12 k\left(\alpha h+k^{1 / 2}\right)} \frac{\mathrm{d} h}{\mathrm{~d} t} .
$$

Equation (A.7) is very similar to the corresponding equation (3.2) in the axisymmetric case. In particular, in the special case of constant load $L=L_{0}>0$ equation (3.2) can be recovered from (A.7) simply by rescaling $t$ with $3 \pi / 16$, and hence the solutions for the fluid layer thickness $h=h(t)$ and the contact time $t_{\mathrm{c}}$ in the two-dimensional case may be readily obtained from the corresponding results in the axisymmetric case.

The path $(x, z)$ taken by a fluid particle is governed by

$$
\begin{aligned}
\frac{1}{x} \frac{\mathrm{d} x}{\mathrm{~d} h} & =-\frac{6(h-z)\left[\left(\alpha h+k^{1 / 2}\right) z+k^{1 / 2}\left(h+2 \delta \alpha k^{1 / 2}\right)\right]}{h^{2}\left(\alpha h^{2}+4 k^{1 / 2} h+6 \delta \alpha k\right)+12 k\left(\alpha h+k^{1 / 2}\right)} \\
\frac{\mathrm{d} z}{\mathrm{~d} h} & =\frac{2\left(\alpha h+k^{1 / 2}\right)\left(6 k-z^{3}\right)+3 \alpha\left(h^{2}-2 \delta k\right) z^{2}+6 k^{1 / 2} h\left(h+2 \delta \alpha k^{1 / 2}\right) z}{h^{2}\left(\alpha h^{2}+4 k^{1 / 2} h+6 \delta \alpha k\right)+12 k\left(\alpha h+k^{1 / 2}\right)}
\end{aligned}
$$

in the fluid layer $0 \leqslant z \leqslant h$, and

$$
\begin{aligned}
\frac{1}{x} \frac{\mathrm{d} x}{\mathrm{~d} h} & =-\frac{12 k\left(\alpha h+k^{1 / 2}\right)}{\phi\left[h^{2}\left(\alpha h^{2}+4 k^{1 / 2} h+6 \delta \alpha k\right)+12 k\left(\alpha h+k^{1 / 2}\right)\right]} \\
\frac{1}{1+z} \frac{\mathrm{d} z}{\mathrm{~d} h} & =\frac{12 k\left(\alpha h+k^{1 / 2}\right)}{\phi\left[h^{2}\left(\alpha h^{2}+4 k^{1 / 2} h+6 \delta \alpha k\right)+12 k\left(\alpha h+k^{1 / 2}\right)\right]}
\end{aligned}
$$

in the porous bed $-1 \leqslant z \leqslant 0$. Hence the particle paths in the porous bed are given by

$$
\frac{x}{x_{0}}=\frac{1+z_{0}}{1+z}=\exp \left[-\frac{12 k}{\phi} \int_{d}^{h} \frac{\alpha s+k^{1 / 2}}{s^{2}\left(\alpha s^{2}+4 k^{1 / 2} s+6 \delta \alpha k\right)+12 k\left(\alpha s+k^{1 / 2}\right)} \mathrm{d} s\right],
$$

and so, in particular, always satisfy $x(1+z)=x_{0}\left(1+z_{0}\right)=$ constant. Equations (A.9) and (A.11), which govern the vertical position $z=z(t)$ of a fluid particle, are identical to the corresponding equations (3.15) and (3.17) in the axisymmetric case, and hence the solutions for $z=z(t)$ and $z_{\text {pen }}$ in the twodimensional case may be immediately obtained from the corresponding solutions in the axisymmetric case. Moreover, the right-hand sides of equations (A.8) and (A.10), which govern the horizontal position $x=x(t)$ of a fluid particle, differ from the right-hand sides of the corresponding equations (3.14) and (3.16) for $r=r(t)$ in the axisymmetric case by only a factor of 2, and so the solution for $x=x(t)$ in the two-dimensional case may be readily obtained from the solution for $r=r(t)$ in the axisymmetric case by using the simple relationship $x / x_{0}=\left(r / r_{0}\right)^{2}$.

\section{B. Appendix B: Limit of Large Permeability $k \rightarrow \infty$}

In this section asymptotic solutions for the fluid layer thickness $h=h(t)(\S \mathrm{B} .1)$ and the fluid particle paths $(r, z)(\S \mathrm{B} .2)$ in the limit of large permeability $k \rightarrow \infty$ are obtained.

In this limit it will transpire that the contact time $t_{\mathrm{c}}=O\left(k^{-1}\right) \ll 1$ is small, and, unlike in the limit of small permeability $k \rightarrow 0$ described in $\S 4$, a regular perturbation expansion for $h(t)$ in powers of $k^{-1 / 2} \ll 1$ is uniformly valid up to the contact time, making this limit somewhat easier to analyse. 


\section{B.1 Asymptotic Solution for $h=h(t)$}

Since the contact time $t_{\mathrm{c}}=O\left(k^{-1}\right)$ we re-scale time $t$ according to

$$
t=k^{-1} T
$$

where $T$ is $O(1)$ in the limit $k \rightarrow \infty$. Seeking a regular perturbation solution to (3.2) with $L=L_{0}$ of the form

$$
h(T)=h_{0}(T)+k^{-1 / 2} h_{1}(T)+O\left(k^{-1}\right),
$$

at $O(1)$ and $O\left(k^{-1 / 2}\right)$ we have

$$
\begin{aligned}
& \frac{\mathrm{d} h_{0}}{\mathrm{~d} T}=-\frac{8 L_{0}}{\pi}, \\
& \frac{\mathrm{d} h_{1}}{\mathrm{~d} h_{0}}=\frac{\delta \alpha h_{0}^{2}}{2},
\end{aligned}
$$

respectively. Solving (B.3) and (B.4) subject to the initial conditions $h_{0}(0)=d$ and $h_{1}(0)=0$ yields the solutions

$$
\begin{gathered}
h_{0}=d-\frac{8 L_{0} T}{\pi}, \\
h_{1}=-\frac{\delta \alpha\left(d^{3}-h_{0}^{3}\right)}{6} .
\end{gathered}
$$

Note that, unlike in the limit of small permeability $k \rightarrow 0$, the effect of permeability appears at leading order and the leading order solution $h_{0}$ given by (B.5) becomes zero at the finite time $T=T_{\mathrm{c}}=\pi d / 8 L_{0}$. Figures 2(a) and 3(b) compare the leading order large- $k$ asymptotic solution for $h=h(t)$, i.e.

$$
h=d-\frac{8 L_{0} k t}{\pi}
$$

with the exact solution for $t=t(h)$ given by (3.8). The contact time $t=t_{\mathrm{c}}$ has the asymptotic expansion

$$
t_{\mathrm{c}}=\frac{\pi d}{8 L_{0} k}-\frac{\pi \delta \alpha d^{3}}{48 L_{0} k^{3 / 2}}+O\left(k^{-2}\right)
$$

Figure 2(b) compares the leading order large- $k$ asymptotic solution for $t_{\mathrm{c}}$, i.e.

$$
t_{\mathrm{c}}=\frac{\pi d}{8 L_{0} k},
$$

with the exact solution given by (3.11). As for the corresponding result in the limit of small permeability $k \rightarrow 0$ given by (4.31), the Beavers-Joseph constant $\alpha$ appears at first order in the large- $k$ asymptotic expansion for $t_{\mathrm{c}}$ given by (B.8), but inspection of the first order term reveals that increasing the slip length $l_{\mathrm{s}}$ now increases (rather than decreases) the contact time $t_{\mathrm{c}}$, consistent with the behaviour shown in Figure 3(b). However, unlike in the limit of small permeability $k \rightarrow 0$, the asymptotic expansion for $t_{\mathrm{c}}$ given by (B.8) is not uniformly valid in the limit $\alpha \rightarrow \infty$ (i.e. in the special case of zero slip length, $\left.l_{\mathrm{s}}=0\right)$ corresponding to the boundary condition (2.9), and so this special case is treated separately by Knox (2013). 


\section{B.2 Asymptotic Solution for the Fluid Particle Paths}

B.2.1 Fluid Particles Initially Situated in the Fluid Layer $z_{0}>0 \quad$ From (3.14) and (3.15) the leading order equations for the path of a fluid particle in the fluid layer are simply

$$
\begin{aligned}
& \frac{\mathrm{d} r}{\mathrm{~d} h}=0 \\
& \frac{\mathrm{d} z}{\mathrm{~d} h}=1
\end{aligned}
$$

Solving (B.10) and (B.11) subject to (3.18) yields

$$
r=r_{0} \quad \text { and } \quad z=h-h^{*}
$$

where $h^{*}=d-z_{0}$, showing that (since $v_{z}=O(k) \gg 1$ is larger than $v_{r}=O\left(k^{1 / 2}\right) \gg 1$ ) at leading order the particle moves vertically downwards through the fluid layer, reaching the interface $z=0$ when $h=h^{*}$ corresponding to $T=T^{*}=\pi z_{0} / 8 L_{0}$, which from (B.1) corresponds to the time

$$
t=t^{*}=\frac{\pi z_{0}}{8 L_{0} k}
$$

In particular, this means that the leading order large- $k$ asymptotic approximation to the curve $r^{*}=1$ is simply the straight line $r_{0}=1$. Figure 7 compares the straight line $r_{0}=1$ with the exact solution for $r^{*}=1$ for $k=10$.

From (3.16) and (3.17) the leading order equations for the path of a fluid particle after it has entered the porous bed are

$$
\begin{aligned}
\frac{1}{r} \frac{\mathrm{d} r}{\mathrm{~d} h} & =-\frac{1}{2 \phi}, \\
\frac{1}{1+z} \frac{\mathrm{d} z}{\mathrm{~d} h} & =\frac{1}{\phi} .
\end{aligned}
$$

Solving (B.14) and (B.15) subject to the initial conditions $r=r_{0}$ and $z=0$ at $h=h^{*}$ yields

$$
\begin{aligned}
& r=r_{0} \exp \left(\frac{h^{*}-h}{2 \phi}\right), \\
& z=-1+\exp \left(-\frac{h^{*}-h}{\phi}\right)
\end{aligned}
$$

for $0 \leqslant h<h^{*}$. In particular, setting $h=0$ in (B.17) shows that the leading order asymptotic solution for $z_{\text {pen }}$ is

$$
z_{\text {pen }}=-1+\exp \left(-\frac{h^{*}}{\phi}\right)
$$

which, in contrast to the corresponding result in the limit of small permeability $k \rightarrow 0$ given by (4.49), depends on $z_{0}$ (via $h^{*}$ ) but is independent of $k$. Figure 6(c) compares the fluid particle paths $(r, z)$ with the leading order large- $k$ asymptotic solution given by (B.12), (B.16) and (B.17). Figure 8(b) compares the leading order large- $k$ asymptotic solution for $z_{\text {pen }}$ given by (B.18) with the exact solution. 
B.2.2 Fluid Particles Initially Situated in the Porous Bed $z_{0}<0 \quad$ A similar analysis for fluid particles initially situated in the porous bed (i.e. with $z_{0}<0$ ) yields

$$
\begin{aligned}
r & =r_{0} \exp \left(\frac{d-h}{2 \phi}\right), \\
z & =-1+\left(1+z_{0}\right) \exp \left(-\frac{d-h}{\phi}\right), \\
z_{\text {pen }} & =-1+\left(1+z_{0}\right) \exp \left(-\frac{d}{\phi}\right) .
\end{aligned}
$$

Figure 6(c) compares the fluid particle paths $(r, z)$ with the leading order large- $k$ asymptotic solution given by (B.19) and (B.20). Figure 8(d) compares the leading order large- $k$ asymptotic solution for $z_{\text {pen }}$ given by (B.21) with the exact solution.

\section{REFERENCES}

ACHeson, D.J. (1990) Elementary Fluid Dynamics. Oxford: Oxford University Press.

Ateshian, G.A. (2009) The role of interstitial fluid pressurization in articular cartilage lubrication, J. Biomech. 42, 1163-1176.

Ateshian, G.A. \& Hung, C.T. (2006) The natural synovial joint: properties of cartilage, Proc. Inst. Mech. Eng. Part J: J. Eng. Tribol. 220, 657-670.

BALAZS, E.A. (2009) The role of hyaluronan in the structure and function of the biomatrix of connective tissues, Struct. Chem. 20, 233-243.

BeAVERS, G.S. \& JoSEPH, D.D. (1967) Boundary conditions at a naturally permeable wall, J. Fluid Mech. 30, 197-207.

Cawthorn, C.J. \& Balmforth, N.J. (2010) Contact in a viscous fluid. Part 1. A falling wedge, J. Fluid Mech. 646, 327-338.

FAM, H., BRyAnt, J.T. \& Kontopoulou, M. (2007) Rheological properties of synovial fluids, Biorheology 44, $59-74$.

Hou, J.S., Mow, V.C., LAI, W.M. \& Holmes, M.H. (1992) An analysis of the squeeze-film lubrication mechanism for articular cartilage, J. Biomech. 25, 247-259.

Knox, D.J. (2013) Squeeze-Film Flows, with Application to the Human Knee Joint, Thesis (PhD). University of Strathclyde (in preparation).

LiN, J.R., LU, R.F. \& YANG, C.B. (2001) Derivation of porous squeeze-film Reynolds equations using the Brinkman model and its application, J. Phys. D: Appl. Phys. 34, 3217-3223.

MARoudAS, A. (1976) Balance between swelling pressure and collagen tension in normal and degenerate cartilage, Nature 260, 808-809.

Mow, V.C., KueI, S.C., LAI, W.M. \& Armstrong, C.G. (1980) Biphasic creep and stress relaxation of articular cartilage in compression: theory and experiments, J. Biomech. Eng. 102, 73-84.

Mow, V.C. \& RATCLIFFE, A. (1997) Structure and function of articular cartilage and meniscus, In: V. C. Mow AND W. C. HaYes, eds. Basic Orthopaedic Biomechanics (2nd ed.), Philadelphia: Lippincott-Raven Publishers, 113-177.

Mow, V.C. \& GUO, X.E. (2002) Mechano-electrochemical properties of articular cartilage: their inhomogeneities and anisotropies, Ann. Rev. Biomed. Eng. 4, 175-209.

NiELD, D.A. (2009) The Beavers-Joseph boundary condition and related matters: a historical and critical note, Transp. Porous Media 78, 537-540.

Nong, K. \& ANDERson, D.M. (2010) Thin film evolution over a thin porous layer: modeling a tear film on a contact lens, SIAM J. Appl. Math. 70, 2771-2795. 
Nuño, N. \& Ahmed, A.M. (2003) Three-dimensional morphometry of the femoral condyles, Clin. Biomech. 18, 924-932.

O'HARA, B.P., URBAN, J.P.G. \& MAROUdAS, A. (1990) Influence of cyclic loading on the nutrition of articular cartilage, Ann. Rheum. Dis. 49, 536-539.

PRAKASH, J. \& VIJ, S.K. (1973) Load capacity and time-height relations for squeeze films between porous plates, Wear 24, 309-322.

PrAKASH, J. \& VIJ, S.K. (1974) Effect of velocity slip on porous-walled squeeze films, Wear 29, 363-372.

SHERwood, J.D. (1990) The hydrodynamic forces on a cylinder touching a permeable wellbore, Phys. Fluids A 2, 1754-1759 (1990).

Skotheim, J.M. \& MAhadevan, L. (2005) Soft lubrication: the elastohydrodynamics of nonconforming and conforming contacts, Phys. Fluids 17, 092101 (2005).

STANDRInG, S. (ed.), (2008) Gray's Anatomy (40th ed.), London: Churchill Livingstone, Chap. 82, 1393-1410.

STEWART, T., Jin, Z.M. \& Fisher, J. (1997) Friction of composite cushion bearings for total knee joint replacements under adverse lubrication conditions, Proc. Inst. Mech. Eng. Part H: J. Eng. Med. 211, 451-465.

Stone, H.A. (2005) On lubrication flows in geometries with zero local curvature, Chem. Eng. Sci. 60, 4838-4845.

SZERI, A.Z. (1998) Fluid Film Lubrication, Cambridge: Cambridge University Press.

Walker, P.S., Yildirim, G., Arno, S. \& Heller, Y. (2010) Future directions in knee replacement, Proc. Inst. Mech. Eng. Part H: J. Eng. Med. 224, 393-414.

WeEkLEY, S.J., WATERS, S.L. \& Jensen, O.E. (2006) Transient elastohydrodynamic drag on a particle moving near a deformable wall, Q. J. Mech. Appl. Math. 59, 277-300.

WU, H. (1970) Squeeze-film behaviour for porous annular disks, J. Lubr. Technol. 92, 593-596.

WU, H. (1972) Effect of velocity-slip on the squeeze film between porous rectangular plates, Wear 20, 67-71. 